Harvard Data Science Review • Issue 2.4, Fall 2020

\title{
Forecasting the 2020 U.S. Elections With Decision Desk HQ: Methodology for Modern American Electoral Dynamics
}

Kiel Williams ${ }^{1}$ Mukul Ram $^{1}$ Matthew Shor ${ }^{1}$ Sreevani Jarugula ${ }^{1}$ Dan DeRemigi ${ }^{1}$ Alex Alduncin ${ }^{1}$ Scott Tranter ${ }^{1}$

${ }^{1} \varnothing p t i m u s$ Analytics, District of Columbia, United States of America

Published on: Oct 27, 2020

DOI: https://doi.org/10.1162/99608f92.9663befd

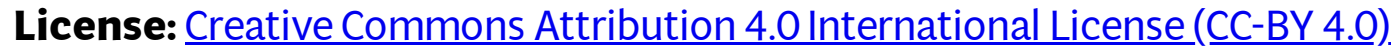




\section{ABSTRACT}

Øptimus has constructed models to predict the outcomes of the 2020 presidential and congressional general elections in collaboration with Decision Desk HQ. The model is an iteration from its 2018 U.S. Congressional model designed to predict the outcome of the election as if it were held today. The congressional model predicts the probability of a Republican (GOP) victory in individual House and Senate elections, as well as the number of aggregate seats expected to be won by each party (to predict partisan control of each chamber). The presidential model uses a similar framework to estimate vote shares and probabilities of victory for each major party candidate in each of the states. $\underline{1}$ These estimates are then used to proxy electoral college predictions that determine who is elected as the next President of the United States. We provide a survey of features, feature engineering techniques, models, and ensembling techniques. We also provide some empirical results.

Keywords: elections, political science, government, machine learning

\section{Media Summary}

We start with a data set of 200+ base features spanning economic indicators, political environment measures (both national and local), candidate traits, campaign finance reports, and engineered variables designed to draw context-specific information into the model. This data set is refreshed on a rolling basis. Not every feature makes it into every model; a large number of these features are most fruitful when paired with certain other features and models. We spend a considerable amount of effort engineering features that reflect some aspect of historic election outcomes that are not quite captured in the raw data. Then we pair features with models (both manually and automatically), and build a set of base models. Occasionally, these base models have target variables that are slightly different from our final dependent variables. We then ensemble these base models together, either by taking a weighted average of the prediction of each model, or by applying a stacking classifier on top of the base model. These predictions are then blended together with probabilities derived from current polling. Using these poll-informed predictions, we run 14,000,605 simulations for the Senate/House forecasts and 140,605 simulations for the Presidential forecast to determine the range of possible outcomes.

We're constantly iterating on our modeling workflow - trying out new features, different ensembling techniques, and configurations. It is likely that our model will exist in a slightly different form by the end of the 2020 election cycle.

\section{Target Variables}

Our model attempts to accurately forecast the outcome of both the US Presidential and Congressional elections in the 2020 election cycle. In both the US Senate and House of Representatives, we provide a probability of 
each party's winning each particular seat. Using these seat-by-seat probabilities, we provide an overall probability of each party winning control of each chamber. We adopt a similar approach for the Presidential election. Our model estimates the likelihood of each candidate winning each state. Using these state-by-state probabilities as a starting point, we simulate possible outcomes in the Electoral College. From these simulations, we derive an overall probability of victory for each candidate.

\section{Data}

There are two broad classes of features in our model: raw and engineered features. $\stackrel{2}{2}$ Raw features are external data fed directly to the model, while engineered features are raw data modified in some way to be more useful to the model. Both categories span various domains, including candidate fundraising, demographic information, economic indicators, electoral history, and political environment.

Many of the features we incorporate are broadly recognized in the political science sphere and beyond. For example, it is well understood that congressional candidates of the President's party are strongly impacted by presidential approval ratings (Edwards, 2009). Demographic variables also fall into this category. The political dynamics of a locality depend strongly on the African-American share of the total population for instance.

In addition to primary source data, we also engineer several features. For instance, in addition to routine financial data provided by the Federal Election Commission (FEC), we also incorporate a formula to compare GOP and Democratic campaign finance numbers in each district/state, as well as an indicator for whether a House race has surpassed \$3 million in contributions or a Senate race has surpassed \$20 million. These thresholds are derived from an internal empirical analysis of what counts as an 'expensive race.' Races that cross this fundraising threshold are typically the most competitive, and are governed by different dynamics than less competitive races. Polling data for each race is consolidated using a weighted average that accounts for recency, sample size and pollster quality. Other engineer features take into account not the total money raised by each candidate, but also level-off as one candidate surpasses the other in fundraising. This incorporates the well-known political science insight that additional fundraising only provides an additional benefit up to a certain point (e.g., Barutt and Schofield, 2016).

We refresh the data set on a rolling basis to ensure that any and all changes to individual races are accounted for quickly. This includes adding any new individual race polling, changes in the national environment and special election environment variables, quarterly and 48-hour FEC reports, new economic indicators, primary election outcomes, and candidate status changes.

\section{Feature Selection}

Our approach strikes a balance between explanatory power and predictive power. The structure of our model is such that we can not only accurately determine the winner of a congressional race for example, but we can also identify what features provided to the model are driving the projected outcome. The success of our model is 
demonstrated by its success during the 2018 midterm elections, when we successfully predicted the outcome in 97\% of House races.

To this end, our final ensemble model is composed of base models whose features are drawn from different sources. The feature sets in some models emerge from political science theory, while others are derived from a more strictly machine-learning driven approach. This approach bolsters the interpretability of our model, and reduces the risk of overfitting. With these concerns in mind, we explicitly avoid a 'kitchen sink' approach to feature selection: including every possible feature may provide a small gain to model accuracy, but only at the cost of model interpretability. There are three key elements of our feature selection approach -

1. Starting with an approach informed by political science literature, we hand-pick a set of features. Academic models predicting congressional results span the latter half of the 20th century (e.g. Lewis-Beck and Rice, 1984; Stokes and Miller, 1962; Tufte, 1975) with relatively simple quantitative analyses, and are still relevant and accurate through the work of contemporary scholars (e.g. Campbell, 2010; Lewis-Beck and Tien, 2014). We chose to include many of the same variables that these researchers find most important, such as incumbency (Abramowitz, 1975; Erikson, 1971), district partisanship (Brady et al., 2000), and whether a given year is a midterm or presidential cycle (Erikson, 1988; Lewis-Beck and Tien, 2014). At the same time, we exclude minor variables that lack a strong theoretical grounding. For example, FEC reports include detailed information about offsets to campaign expenditures, and refunded individual campaign contributions. These variables — alongside many others provided by the FEC—do not provide the model with useful new information, beyond what is already captured by a campaign's overall fundraising. As a result, we exclude variables like these from the feature set. We do include a ratio of GOP and Democratic contributions to incorporate FEC data at large because, while scholars typically fail to find a general causal linkage between raising more money and winning, it does appear to be a considerably predictive variable for challenger success (Jacobson, 1978).

2. Beginning from this feature set derived from the political science literature, we conduct feature selection by determining linear dependence of features with one another and weeding out variables that are highly correlated. We use ANOVA F-values in order to determine variance between features (Pedregosa et al., 2011).

3. Finally—leaving behind the explanatory power afforded by political science theory—we conduct a randomized feature selection and optimize over accuracy and ROC-AUC (Receiver Operating Characteristics-Area Under Curve) (Zou and Hastie, 2003). We use ridge and lasso regression in this stage (the elastic net approach).

After determining the relevant feature sets, we pair them with various models and back-test them to see which feature-model pairs are ideal. Example pairings might include a Random Forest model paired with features generated via elastic net feature selection, and a logistic regression using hand-picked feature set. There is a good deal of caution applied at this stage in order to ensure that we are not merely overfitting to historical data: 
a model tailored too closely to the 2016 election may not perform well in another year, for instance. In this manner, our final predictions incorporate information from the best features identified by both political science and machine-learning, while mitigating the shortcomings of each approach.

\section{Model Choice}

Most election forecasting models use either a Bayesian or frequentist approach to predict the outcome of an election. We find that empirically, both perform quite well and have different strengths with respect to inference. Because different models complement one another in this way, our modeling process adopts an ensemble approach, incorporating different kinds of models including Bayesian logistic regressions, logistic regressions, Random Forests, XGBoosts, and Elastic Nets. Because of the quantity of congressional data available to us - 435 races every two years extending back to 1992 in the House of Representatives, for example - the prior for a Bayesian regression is not very significant, and the regression performs similarly to one conducted in a frequentist framework. The predictions produced by each model associated feature set are then averaged together into the final overall ensemble prediction. While more sophisticated ensembling algorithms based on model 'boosting' are well-known in the literature, our simpler approach accurately predicts the outcome in 95\% of 2018 congressional election. The individual models composing the ensemble produce equivalent accuracies between 90 and 95\%.

Including a variety of models and variable subsets in our ensemble reduces error in two ways. First, ensembles have proven to be more accurate on average than their constituent models alone. Second, they are less prone to making substantial errors (i.e., if they miss, they miss by smaller margins on average); see Montgomery et al., 2012. Individual models produce good results, but give different estimates for each race. Individual models typically produce similar accuracy and F1 scores, but produce better estimates when averaged together. Our empirical results from 2018 illustrate the success of this approach.

In the House model, we combine two separate ensemble models— one based on candidate party affiliation, and the other based on incumbency — and then add recent polling information. In the Senate, a single party-oriented ensemble model is sufficient to produce accurate results, and is later combined with polls to make a final prediction.

In the Presidential model, we adopted a different approach from the House and Senate. This is a result of data availability: usable historical data for the presidency extends back only to 1992. This time window encompasses only seven Presidential elections on which to train a model. This makes Presidential models particularly prone to overfitting. Combine this with the fact that the national environment is extraordinarily volatile, and one has a recipe for uncertainty. We overcame this problem by implementing a stacking ensemble that incorporates a collection of different submodels. Because these constituent models differ in both feature set and model-type (logit, SVM, Random Forest, and XGBoost), we were able to avoid severe overfitting, 
given the limited amount of training data available. This approach back-tested better than any of the alternatives, especially with regards to model calibration.

\section{Polls}

Poll results are a key ingredient in our model. Each individual poll in a race is converted to a probability representing the likelihood of a GOP win. This probability is generated by sampling from a posterior normal distribution centered on the share of the vote GOP received by the Republican candidate in a particular poll. The variance $V$ of the normal distribution is determined predominantly by the sample size of the poll, and the typical methodology of the pollster. We simulate election outcomes from each poll by drawing a GOP vote share $R$ from the resulting normal distribution:

$$
R \sim N(G O P, V)
$$

From this simulated Republic vote share $R$, we simulate a Democratic vote share $D$ as:

$$
D=G O P+D E M-R
$$

where DEM is the Democratic vote share reported by the poll. By comparing each set of simulated vote shares, we determine a probability of Republican victory. If the GOP candidate's vote share is greater than that of their Democratic opponent, a GOP win is recorded. The number of GOP wins divided by the total number of draws represents a simulated probability of a GOP win, given the poll's margin.

Public polls make up the bulk of polling in our model. For clients, we commission private polling with turnout modeling and consistent data collection methodology. Because private polls typically have larger sample sizes than public polls - and are typically concentrated in key battleground states - they play a significant role in improving our model performance. Private polls also frequently sample individuals using a registration-based (RBS) methodological approach, in contrast to the random digit dialing (RDD) often used in public polling. Existing literature has found polls based on RBS to often provide more accurate results in congressional races (Green and Gerber, 2006).

The spread of the sampling distribution is based on the estimated total survey error of the poll. Since a poll's reported margin of error often does not adequately capture its uncertainty (Shirani-Mehr et al., 2018), we perform an adjustment to better reflect the true uncertainty of a GOP win. Using an empirical distribution of polling errors gathered from House and Senate races dating back to 2006 as a baseline, we adjust the margin of error associated with each poll. These adjustments vary by poll, and depend on both the methodology of a poll, and its proximity to the election. The margin of error on higher-quality polls, and on polls conducted closer to the election, are adjusted downward. The typical pollster-based margin of error adjustment is approximately 20$30 \%$. The individual probabilities are then ensembled. 
Weights are based on a poll's proximity to the election, as well as to the pollster's FiveThirtyEight rating. A linear decay function is applied to the poll's date as well as the polls rating. Polls with higher pollster ratings that are closer to the election are weighted more heavily. The final probability we project for a given race is a weighted average between the poll and non-poll probabilities, with the weight of the poll probability increasing as the election becomes closer in time. Polling weights were developed by Øptimus during the 2018 election cycle, and were successful in back-testing on previous election cycles. The approach incorporates well-known insight from political science regarding the reliability of polls at different points in the election cycle. Our method also allows the model to separate lower-quality polls from those likely to be higher in quality.

\section{Election Simulations}

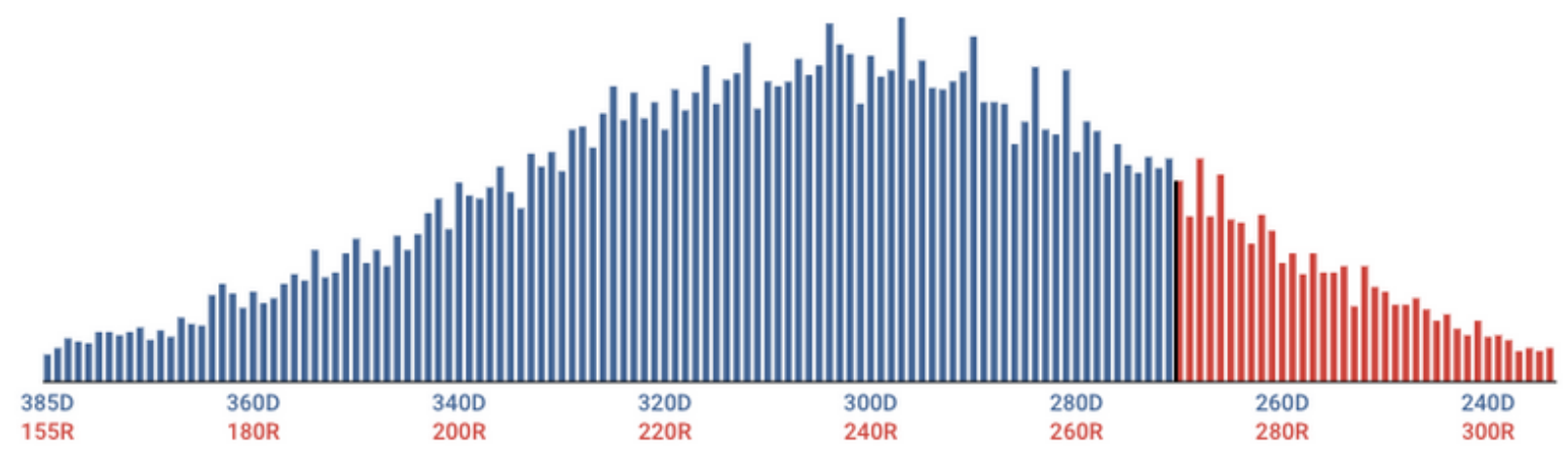

Figure 1. A simulated distribution of electoral college outcomes using the modeling approach we describe. As of this writing (Summer 2020), we project Donald Trump to receive an average of 235 electoral votes to 303 for Joe Biden. Joe Biden defeats Donald Trump $84 \%$ of the time.

Using the computed probabilities for each House, Senate, and Presidential race, we predict the aggregate number of seats we expect the GOP to win and the probability of maintaining control of the House and Senate. We use each seat's predicted probabilities to run simulations of the 2020 Congressional elections.

The final outcomes in different races are strongly correlated with one another. In 2016 for example, we saw this occur in the upper midwest: Trump not only outperformed in Wisconsin, but also in states like Michigan, Iowa, and Pennsylvania that share similar demographic profiles. Polling errors are mildly correlated across races within an election cycle due to various sources of error, which can result in systematic bias. However, across election cycles going back several decades, the mean partisan bias computed over all polls is approximately zero (Shirani-Mehr et al., 2018). Because the overall partisan bias of polling in a given year is not a priori known, this is not explicitly corrected for within our model.

The mechanism of a wave election - an election in which one party performs overwhelmingly better than the other-is simulated by treating our predicted probabilities as beta random variables. Each race is assigned a beta distribution centered on the predicted probability, with shape parameters chosen to reflect the volatility of 
toss-up races in wave elections and conversely, the relative resilience of non-competitive races. Within a given simulation, as the outcome in each state is sequentially determined, the probability of victory for each party in each remaining state is modified in reaction. Thus—as a candidate rises or falls in a particular simulationtheir fortunes elsewhere rise or fall. In this manner, state-to-state correlations are explicitly incorporated into our simulation framework.

We perform over 10 million simulations to create a distribution of potential outcomes. This approach allows us to qualitatively analyze individual 'scenarios' for a more narrative backed description of how the election will turn out. For example, we can find the most likely path to victory for a candidate, contingent on them winning or losing in a specific set of states.

For the Presidential race, we draw from a Binomial distribution for each state and then calculate electoral college totals in order to determine the overall distribution of electoral votes. Attempts to force certain correlations between states did not produce significantly different simulation results.

\section{Empirical Results}

This cycle's model $\underline{3}$ is an iteration of a model we released in 2018. In 2018, we publicly released our House and Senate predictions beginning in June and updating regularly until Election Day. Our final House prediction had Democrats at a $95.9 \%$ chance of taking control of the chamber. The mean prediction was 233 Democratic seats to 202 GOP seats, and the 90\% confidence interval spanned from 218 to 248 Democratic seats. Control of the House was called early in the night by most outlets. Ultimately, Democrats won 235 seats, and Republicans won 200. This outcome produced an overall accuracy of $97 \%$ for our model, with predictions within the $90 \%$ confidence interval of the model typically producing an accuracy between $93 \%$ and $100 \%$.

Because congressional incumbents are overwhelmingly reelected, a good baseline for comparison is provided by simply assuming that incumbents are all reelected, and retirements result in no change of partisan control. This simplistic model would incorrectly predict the outcome in 45 US House races held during the 2018 election, producing an overall accuracy of $90 \%, 7 \%$ worse than our model. The difference is even more stark when we only examine the 31 House seats we identified as toss-up. Among this subset, our model achieves a $67 \%$ accuracy, while simply assuming incumbent victory would result in only a 32\% accuracy. Because House control is determined largely by the outcomes in these kinds of competitive races, this may be a better baseline for comparison.

On the Senate side, our final prediction gave Republicans a 91.9\% chance of keeping control of the chamber. The mean seat prediction was 52 GOP seats to 48 Democratic seats, with a 90\% confidence interval spanning from 49 GOP seats to 55 GOP seats. Our GOP chance of keeping the majority peaked above $89 \%$ at three different points: in mid-August, in mid-October, and right before the election. As with the House race, chamber control was decided early. The final outcome in the Senate was 53 GOP seats to 47 Democratic seats. Among all 35 Senate seats, our model correctly predicted 33, for an overall accuracy of 94\%. In contrast, a baseline 
model assuming incumbent-party victory would have incorrectly forecast 6 Senate races that changed partisan control, for an accuracy of only $83 \%$.

Table 1 contains individual race performance metrics for the Øptimus House 2018 model. For the 434 races called ${ }^{4}$, the Øptimus House model called 421/434 races correctly, or an accuracy measure of $96.8 \%$. Among the 31 toss-ups, the model predicted 21/31 toss-up races correctly, meaning it had 67.7\% accuracy among these races. Excluding the toss-ups, the House model predicted 400 out of 404 non-toss-up races correctly, or 99.01\% accuracy. The orientation of the metrics is based on the Republican win percentage. A true positive is a correctly predicted Republican victory, while a false positive is a predicted Republican victory that was actually a Democratic win.

Table 1. 2018 House model performance.

\begin{tabular}{|c|c|c|c|}
\hline & All Seats & Excluding Toss-Ups & Toss-Ups Only \\
\hline Number of Seats & 434 & 403 & 31 \\
\hline Accuracy & $97.00 \%$ & $99.26 \%$ & $67.74 \%$ \\
\hline Total Misses & 13 & 3 & 10 \\
\hline False Negatives & 3 & 0 & 3 \\
\hline False Positives & 10 & 3 & 7 \\
\hline True Negatives & 225 & 210 & 15 \\
\hline True Positives & 196 & 190 & 6 \\
\hline Brier Score & 0.034 & 0.019 & 0.235 \\
\hline Matthew's Correlation & 0.94 & 0.985 & 0.321 \\
\hline AUC & 0.996 & 0.998 & 0.763 \\
\hline $\mathbf{F 1}$ & 0.968 & 0.992 & 0.546 \\
\hline $\mathbf{F} 2$ & 0.978 & 0.997 & 0.612 \\
\hline Precision & 0.952 & 0.985 & 0.462 \\
\hline Recall & 0.985 & 1 & 0.667 \\
\hline
\end{tabular}


Table 2 contains the performance scores for the Øptimus Senate model. The Øptimus Senate model predicted 33 out of 35 races correctly, an accuracy measure of $94.29 \%$. Among the 4 toss-ups called, the model correctly predicted 3 out of 4 races. Out of the 31 non-toss-ups, the only race missed by the Senate model is the Florida Senate seat. As with Table 1, the orientation of the metrics is based on the Republican win percentage.

Table 2. 2018 Senate model performance.

\begin{tabular}{|c|c|c|c|}
\hline & All Seats & Excluding Toss-Ups & Toss-Ups Only \\
\hline Number of Seats & 35 & 31 & 4 \\
\hline Accuracy & $94.29 \%$ & $96.77 \%$ & $75.00 \%$ \\
\hline Total Misses & 2 & 1 & 1 \\
\hline False Negatives & 2 & 1 & 1 \\
\hline False Positives & 0 & 0 & 0 \\
\hline True Negatives & 24 & 22 & 2 \\
\hline True Positives & 9 & 8 & 1 \\
\hline Brier Score & 0.056 & 0.031 & 0.246 \\
\hline Matthew's Correlation & 0.869 & 0.922 & 0.577 \\
\hline AUC & 0.985 & 1.000 & 0.500 \\
\hline F1 & 0.9 & 0.941 & 0.667 \\
\hline F2 & 0.849 & 0.909 & 0.556 \\
\hline Precision & 1.000 & 1.000 & 1.000 \\
\hline Recall & 0.818 & 0.889 & 0.500 \\
\hline
\end{tabular}

Table 3 (House) and 4 (Senate) display the accuracy and total number of incorrect predictions made by every individual model included in our ensemble in 2018, as well as by the final ensemble of individual models and polls. In this table, we note the performance of each individual model included in the ensemble: Random Forests, logits, XGBoost, Bayesian MCMC, and the corresponding ensemble performance. The Bayesian MCMC calculations were performed using the JAGS and PyMC3 computational packages. 
Models using the 'Poli Sci' feature set rely upon a set of features widely regarded as crucial variables in political science literature. The 'Select K Best' feature sets are determined algorithmically, using ANOVA Fvalues to minimize collinearity between all features included in the set. The 'Elastic Net' feature sets are similarly produced, using the elastic net approach to algorithmically identify the best features to include.

As expected, the ensemble performs better on average than the individual models that compose the ensemble. For example, in Table 3 the main ensemble model incorrectly predicted only 21 of the 434 called House tossup races in 2018, in contrast to 22-41 misses each for the individual models composing the ensemble. While some individual models outperform the overall ensemble with respect to accuracy, most do not. Because there is no way to determine a priori which constituent models will outperform, the ensemble remains the best overall choice. Creating an ensemble of individual models helps to minimize the systematic bias in each of the models. In this way, weaknesses of individual models can be compensated by combining them together

The final rows of both Table 3 and 4 indicate the performance of the ensemble when combined with polling data. A linear combination of the chances of GOP victory based on ensemble of individual models and polls gives the best performing model. We have observed this in our out-of-sample back-tests (2016, 2014, 2010, and 2006) as well. For example, the inclusion of polls into the 2018 House ensemble boosts accuracy by around 2\%, while polling data boosts accuracy of the 2018 Senate ensemble by $8 \%$. Because polling is typically more prevalent in the Senate than in the House, it is unsurprising that polling does more to improve the Senate model.

Table 3. 2018 House performance by model.

\begin{tabular}{|l|l|l|l|l|l|l|l|l|l|}
\hline \multicolumn{2}{|l|}{$\begin{array}{l}\text { UNDERLYING } \\
\text { MODELS }\end{array}$} & \multicolumn{2}{|l|}{ ALL SEATS (434) } & \multicolumn{2}{l|}{ NON TOSS UPS (403) } & \multicolumn{2}{l|}{ TOSS UPS (31) } \\
\hline $\begin{array}{l}\text { FEATURE } \\
\text { SELECTI } \\
\text { ON }\end{array}$ & MODEL & $\begin{array}{l}\text { NUMBER } \\
\text { OF } \\
\text { VARIABL } \\
\text { ES }\end{array}$ & $\begin{array}{l}\text { ACCURA } \\
\text { CY }\end{array}$ & $\begin{array}{l}\text { TOTAL } \\
\text { MISSES }\end{array}$ & $\begin{array}{l}\text { ACCURA } \\
\text { CY }\end{array}$ & $\begin{array}{l}\text { TOTAL } \\
\text { MISSES }\end{array}$ & $\begin{array}{l}\text { ACCURA } \\
\text { CY }\end{array}$ & $\begin{array}{l}\text { TOTAL } \\
\text { MISSES }\end{array}$ \\
\hline $\begin{array}{l}\text { Select K } \\
\text { Best }\end{array}$ & $\begin{array}{l}\text { Random } \\
\text { Forest }\end{array}$ & 175 & $92.40 \%$ & 33 & $96.77 \%$ & 13 & $35.48 \%$ & 20 \\
\hline Pol Sci & $\begin{array}{l}\text { Logistic } \\
\text { Regression }\end{array}$ & 26 & $94.01 \%$ & 26 & $97.52 \%$ & 10 & $48.39 \%$ & 16 \\
\hline Pol Sci & $\begin{array}{l}\text { Random } \\
\text { Forest }\end{array}$ & 26 & $92.63 \%$ & 32 & $96.53 \%$ & 14 & $41.94 \%$ & 18 \\
\hline
\end{tabular}




\begin{tabular}{|c|c|c|c|c|c|c|c|c|}
\hline $\begin{array}{l}\text { Select K } \\
\text { Best }\end{array}$ & $\begin{array}{l}\text { Bayesian } \\
\text { Modeling - } \\
\text { MCMC( } \\
\text { JAGS) }\end{array}$ & 30 & $94.93 \%$ & 22 & $97.27 \%$ & 11 & $64.52 \%$ & 11 \\
\hline $\begin{array}{l}\text { Select K } \\
\text { Best }\end{array}$ & $\begin{array}{l}\text { Logistic } \\
\text { Regression }\end{array}$ & 31 & $92.63 \%$ & 32 & $95.78 \%$ & 17 & $51.61 \%$ & 15 \\
\hline $\begin{array}{l}\text { Select K } \\
\text { Best }\end{array}$ & $\begin{array}{l}\text { Bayesian } \\
\text { Modeling - } \\
\text { MCMC } \\
\text { (PyMC3) }\end{array}$ & 31 & $94.47 \%$ & 24 & $97.02 \%$ & 12 & $61.29 \%$ & 12 \\
\hline $\begin{array}{l}\text { Select K } \\
\text { Best }\end{array}$ & $\begin{array}{l}\text { Random } \\
\text { Forest }\end{array}$ & 31 & $91.94 \%$ & 35 & $96.03 \%$ & 16 & $38.71 \%$ & 19 \\
\hline $\begin{array}{l}\text { Select K } \\
\text { Best }\end{array}$ & XGBoost & 31 & $94.70 \%$ & 23 & $97.52 \%$ & 10 & $58.06 \%$ & 13 \\
\hline Elastic Net & $\begin{array}{l}\text { Random } \\
\text { Forest }\end{array}$ & 33 & $90.55 \%$ & 41 & $94.29 \%$ & 23 & $41.94 \%$ & 18 \\
\hline $\begin{array}{l}\text { Select K } \\
\text { Best }\end{array}$ & $\begin{array}{l}\text { Logistic } \\
\text { Regression }\end{array}$ & 61 & $91.71 \%$ & 36 & $93.55 \%$ & 26 & $67.74 \%$ & 10 \\
\hline $\begin{array}{l}\text { Select K } \\
\text { Best }\end{array}$ & $\begin{array}{l}\text { Random } \\
\text { Forest }\end{array}$ & 61 & $91.71 \%$ & 36 & $96.03 \%$ & 16 & $35.48 \%$ & 20 \\
\hline $\begin{array}{l}\text { Select K } \\
\text { Best }\end{array}$ & XGBoost & 61 & $94.24 \%$ & 25 & $97.27 \%$ & 11 & $54.84 \%$ & 14 \\
\hline \multicolumn{3}{|c|}{ Main Ensemble Only } & $95.16 \%$ & 21 & $98.01 \%$ & 8 & $58.06 \%$ & 13 \\
\hline Main + Incu & mbency Ense & mble & $95.16 \%$ & 21 & $98.26 \%$ & 7 & $54.84 \%$ & 14 \\
\hline Ensembles + & Polls & & $97.00 \%$ & 13 & $99.26 \%$ & 3 & $67.74 \%$ & 10 \\
\hline
\end{tabular}

Table 4. 2018 Senate performance by model

\begin{tabular}{|l|l|l|l|}
\hline $\begin{array}{l}\text { UNDERLYING } \\
\text { MODELS }\end{array}$ & ALL SEATS & NON TOSS UPS & TOSS UPS \\
\hline
\end{tabular}




\begin{tabular}{|c|c|c|c|c|c|c|c|c|}
\hline $\begin{array}{l}\text { FEATURE } \\
\text { SELECTI } \\
\text { ON }\end{array}$ & MODEL & $\begin{array}{l}\text { NUMBER } \\
\text { OF } \\
\text { VARIABL } \\
\text { ES }\end{array}$ & $\begin{array}{l}\text { ACCURA } \\
\mathrm{CY}\end{array}$ & $\begin{array}{l}\text { TOTAL } \\
\text { MISSES }\end{array}$ & $\begin{array}{l}\text { ACCURA } \\
\mathrm{CY}\end{array}$ & $\begin{array}{l}\text { TOTAL } \\
\text { MISSES }\end{array}$ & $\begin{array}{l}\text { ACCURA } \\
\mathrm{CY}\end{array}$ & $\begin{array}{l}\text { TOTAL } \\
\text { MISSES }\end{array}$ \\
\hline Elastic Net & $\begin{array}{l}\text { Bayesian } \\
\text { Modeling - } \\
\text { PyMC3 }\end{array}$ & 10 & $82.86 \%$ & 6 & $90.32 \%$ & 3 & $25.00 \%$ & 3 \\
\hline Pol Sci & $\begin{array}{l}\text { Logistic } \\
\text { Regression }\end{array}$ & 20 & $80.00 \%$ & 7 & $83.87 \%$ & 5 & $50.00 \%$ & 2 \\
\hline Pol Sci & $\begin{array}{l}\text { Random } \\
\text { Forest }\end{array}$ & 20 & $80.00 \%$ & 7 & $83.87 \%$ & 5 & $50.00 \%$ & 2 \\
\hline $\begin{array}{l}\text { Select K } \\
\text { Best }\end{array}$ & $\begin{array}{l}\text { Logistic } \\
\text { Regression }\end{array}$ & 26 & $80.00 \%$ & 7 & $90.32 \%$ & 3 & $0.00 \%$ & 4 \\
\hline Elastic Net & $\begin{array}{l}\text { Bayesian } \\
\text { Modeling - } \\
\text { JAGS }\end{array}$ & 27 & $80.00 \%$ & 7 & $83.87 \%$ & 5 & $50.00 \%$ & 2 \\
\hline Elastic Net & Elastic Net & & $85.71 \%$ & 5 & $90.32 \%$ & 3 & $50.00 \%$ & 2 \\
\hline \multicolumn{2}{|l|}{ Ensemble } & & $85.71 \%$ & 5 & $90.32 \%$ & 3 & $50.00 \%$ & 2 \\
\hline \multicolumn{2}{|c|}{ Ensembles + Polls } & & $94.29 \%$ & 2 & $96.77 \%$ & 1 & $75.00 \%$ & 1 \\
\hline
\end{tabular}

\section{Acknowledgments}

The authors would like to thank Don Green for his feedback to the forecasting model during its development. Additionally, we are grateful for the essential contributions of Neha Bora and Jakob Grimmius, former Øptimus modeling team members and pioneers of the 2018 forecast. Finally, we would also like to thank Olivia Blute, Austin Kim, and Alexander Podkul for supporting the modeling team in years past and present.

\section{Disclosure Statement}

Every author of this article either is employed, or has recently been employed, by Øptimus Analytics, a data science firm specializing in predictive modeling across the public and private sector. 


\section{Appendix}

Table A1. List of variables used in our models.

\begin{tabular}{|c|c|c|c|c|c|}
\hline Name & House & Senate & President & Description & Source \\
\hline $\begin{array}{l}3 \text { Month Net } \\
\text { Change in Weekly } \\
\text { Wage }\end{array}$ & $\mathrm{T}$ & $\mathrm{T}$ & $\mathrm{F}$ & $\begin{array}{l}\text { Net change in } \\
\text { weekly wage over } \\
\text { previous } 3 \text { months }\end{array}$ & $\begin{array}{l}\text { Federal Reserve } \\
\text { Economic Data }\end{array}$ \\
\hline $\begin{array}{l}3 \text { Month Percent } \\
\text { Change in Weekly } \\
\text { Wage }\end{array}$ & $\mathrm{T}$ & $\mathrm{T}$ & $\mathrm{F}$ & $\begin{array}{l}\text { Percent change in } \\
\text { weekly wage over } \\
\text { previous } 3 \text { months }\end{array}$ & $\begin{array}{l}\text { Federal Reserve } \\
\text { Economic Data }\end{array}$ \\
\hline Adjusted PVI & $\mathrm{T}$ & $\mathrm{T}$ & $\mathrm{F}$ & $\begin{array}{l}\text { PVI+national } \\
\text { environment }\end{array}$ & $\begin{array}{l}\text { Calculated in- } \\
\text { house }\end{array}$ \\
\hline Asian Pct & $\mathrm{F}$ & $\mathrm{F}$ & $\mathrm{T}$ & $\begin{array}{l}\text { Asian population } \\
\text { percent }\end{array}$ & US Census Bureau \\
\hline $\begin{array}{l}\text { Average Weekly } \\
\text { Wage }\end{array}$ & $\mathrm{T}$ & $\mathrm{T}$ & $\mathrm{F}$ & $\begin{array}{l}\text { Average weekly } \\
\text { wage in the } \\
\text { previous quarter }\end{array}$ & $\begin{array}{l}\text { Federal Reserve } \\
\text { Economic Data }\end{array}$ \\
\hline $\begin{array}{l}\text { Bachelor's Degree } \\
\text { Pct }\end{array}$ & $\mathrm{T}$ & $\mathrm{T}$ & $\mathrm{T}$ & $\begin{array}{l}\text { Bachelor's degree } \\
\text { percent }\end{array}$ & US Census Bureau \\
\hline Black Pct & $\mathrm{F}$ & $\mathrm{F}$ & $\mathrm{T}$ & $\begin{array}{l}\text { Black population } \\
\text { percent }\end{array}$ & US Census Bureau \\
\hline CFG Involvement & $\mathrm{T}$ & $\mathrm{T}$ & $\mathrm{T}$ & $\begin{array}{l}\text { CGF spent money } \\
\mathrm{T} / \mathrm{F}\end{array}$ & $\begin{array}{l}\text { Federal Election } \\
\text { Commission }\end{array}$ \\
\hline CFG Percent & $\mathrm{T}$ & $\mathrm{T}$ & $\mathrm{T}$ & $\begin{array}{l}\text { Percent of } \\
\text { spending from } \\
\text { CFG }\end{array}$ & $\begin{array}{l}\text { Federal Election } \\
\text { Commission }\end{array}$ \\
\hline CLF Involvement & $\mathrm{T}$ & $\mathrm{F}$ & $\mathrm{T}$ & $\begin{array}{l}\text { CLF spent money } \\
\mathrm{T} / \mathrm{F}\end{array}$ & $\begin{array}{l}\text { Federal Election } \\
\text { Commission }\end{array}$ \\
\hline CLF Percent & $\mathrm{T}$ & $\mathrm{F}$ & $\mathrm{T}$ & $\begin{array}{l}\text { Percent of } \\
\text { spending from } \\
\text { CLF }\end{array}$ & $\begin{array}{l}\text { Federal Election } \\
\text { Commission }\end{array}$ \\
\hline
\end{tabular}




\begin{tabular}{|c|c|c|c|c|c|}
\hline $\begin{array}{l}\text { Congressional } \\
\text { District or Senate } \\
\text { Class }\end{array}$ & $\mathrm{T}$ & $\mathrm{T}$ & $\mathrm{F}$ & $\begin{array}{l}\text { Congressional } \\
\text { District or Senate } \\
\text { Class }\end{array}$ & $\begin{array}{l}\text { Historical election } \\
\text { results }\end{array}$ \\
\hline CPI & $\mathrm{F}$ & $\mathrm{F}$ & $\mathrm{T}$ & $\begin{array}{l}\text { Consumer price } \\
\text { index }\end{array}$ & $\begin{array}{l}\text { Federal Reserve } \\
\text { Economic Data }\end{array}$ \\
\hline D 2 Party Pct & $\mathrm{F}$ & $\mathrm{F}$ & $\mathrm{T}$ & $\begin{array}{l}\text { Democratic } \\
\text { percentage of two- } \\
\text { party vote }\end{array}$ & $\begin{array}{l}\text { Historical election } \\
\text { results }\end{array}$ \\
\hline $\begin{array}{l}\text { D Candidate } \\
\text { Ideology }\end{array}$ & $\mathrm{F}$ & $\mathrm{F}$ & $\mathrm{T}$ & $\begin{array}{l}\text { Democratic } \\
\text { candidate ideology }\end{array}$ & $\begin{array}{l}\text { Database on } \\
\text { Ideology, Money } \\
\text { in Politics, and } \\
\text { Elections }\end{array}$ \\
\hline $\begin{array}{l}\text { D Consecutive } \\
\text { Terms }\end{array}$ & $\mathrm{F}$ & $\mathrm{F}$ & $\mathrm{T}$ & $\begin{array}{l}\text { Number of } \\
\text { consecutive } \\
\text { Democratic terms }\end{array}$ & $\begin{array}{l}\text { Historical election } \\
\text { results }\end{array}$ \\
\hline D Home State & $\mathrm{F}$ & $\mathrm{F}$ & $\mathrm{T}$ & $\begin{array}{l}\text { Home state of } \\
\text { Democratic } \\
\text { presidential } \\
\text { candidate }\end{array}$ & $\begin{array}{l}\text { Historical election } \\
\text { results }\end{array}$ \\
\hline D IEM Price & $\mathrm{F}$ & $\mathrm{F}$ & $\mathrm{T}$ & $\begin{array}{l}\text { Closing price for } \\
\text { Democratic } \\
\text { candidate in } \\
\text { winner-take-all } \\
\text { market on day } \\
\text { before election }\end{array}$ & $\begin{array}{l}\text { Iowa Electronic } \\
\text { Markets }\end{array}$ \\
\hline $\begin{array}{l}\text { D Incumbent } \\
\text { Candidate }\end{array}$ & $\mathrm{F}$ & $\mathrm{F}$ & $\mathrm{T}$ & $\begin{array}{l}\text { Democratic } \\
\text { incumbent running }\end{array}$ & $\begin{array}{l}\text { Historical election } \\
\text { results }\end{array}$ \\
\hline D Incumbent Party & $\mathrm{F}$ & $\mathrm{F}$ & $\mathrm{T}$ & $\begin{array}{l}\text { Democratic } \\
\text { incumbent running }\end{array}$ & $\begin{array}{l}\text { Historical election } \\
\text { results }\end{array}$ \\
\hline D Overall Pct & $\mathrm{F}$ & $\mathrm{F}$ & $\mathrm{T}$ & $\begin{array}{l}\text { Democratic } \\
\text { percentage of } \\
\text { overall vote }\end{array}$ & $\begin{array}{l}\text { Historical election } \\
\text { results }\end{array}$ \\
\hline
\end{tabular}




\begin{tabular}{|c|c|c|c|c|c|}
\hline $\begin{array}{l}\text { D President Net } \\
\text { Approval }\end{array}$ & $\mathrm{F}$ & $\mathrm{F}$ & $\mathrm{T}$ & $\begin{array}{l}\text { Net approval rating } \\
\text { for Democratic } \\
\text { president }\end{array}$ & $\begin{array}{l}\text { The American } \\
\text { Presidency Project }\end{array}$ \\
\hline D Primary Margin & $\mathrm{F}$ & $\mathrm{F}$ & $\mathrm{T}$ & $\begin{array}{l}\text { Difference in } \\
\text { overall primary } \\
\text { popular vote } \\
\text { percentage } \\
\text { between } \\
\text { Democratic } \\
\text { nominee and } \\
\text { closest primary } \\
\text { challenger }\end{array}$ & $\begin{array}{l}\text { Historical election } \\
\text { results }\end{array}$ \\
\hline D VP Home State & $\mathrm{F}$ & $\mathrm{F}$ & $\mathrm{T}$ & $\begin{array}{l}\text { Home state of } \\
\text { Democratic vice } \\
\text { presidential } \\
\text { candidate }\end{array}$ & $\begin{array}{l}\text { Historical election } \\
\text { results }\end{array}$ \\
\hline D Win & $\mathrm{F}$ & $\mathrm{F}$ & $\mathrm{T}$ & Democratic win & $\begin{array}{l}\text { Historical election } \\
\text { results }\end{array}$ \\
\hline $\begin{array}{l}\text { DCCC } \\
\text { Involvement }\end{array}$ & $\mathrm{T}$ & $\mathrm{F}$ & $\mathrm{F}$ & $\begin{array}{l}\text { DCCC spent } \\
\text { money T/F }\end{array}$ & $\begin{array}{l}\text { Federal Election } \\
\text { Commission }\end{array}$ \\
\hline DCCC Percent & $\mathrm{T}$ & $\mathrm{F}$ & $\mathrm{F}$ & $\begin{array}{l}\text { Percent of } \\
\text { spending from } \\
\text { DCCC }\end{array}$ & $\begin{array}{l}\text { Federal Election } \\
\text { Commission }\end{array}$ \\
\hline Decade & $\mathrm{T}$ & $\mathrm{F}$ & $\mathrm{F}$ & $>2010$ or $<2010$ & $\begin{array}{l}\text { Historical election } \\
\text { results }\end{array}$ \\
\hline Dem CFG Oppose & $\mathrm{T}$ & $\mathrm{T}$ & $\mathrm{T}$ & $\begin{array}{l}\text { Amount spent by } \\
\text { CFG opposing } \\
\text { Democrat }\end{array}$ & $\begin{array}{l}\text { Federal Election } \\
\text { Commission }\end{array}$ \\
\hline Dem CFG Support & $\mathrm{T}$ & $\mathrm{T}$ & $\mathrm{T}$ & $\begin{array}{l}\text { Amount spent by } \\
\text { CFG supporting } \\
\text { Democrat }\end{array}$ & $\begin{array}{l}\text { Federal Election } \\
\text { Commission }\end{array}$ \\
\hline
\end{tabular}




\begin{tabular}{|c|c|c|c|c|c|}
\hline Dem CLF Oppose & $\mathrm{T}$ & $\mathrm{F}$ & $\mathrm{T}$ & $\begin{array}{l}\text { Amount spent by } \\
\text { CLF opposing } \\
\text { Democrat }\end{array}$ & $\begin{array}{l}\text { Federal Election } \\
\text { Commission }\end{array}$ \\
\hline Dem CLF Support & $\mathrm{T}$ & $\mathrm{F}$ & $\mathrm{T}$ & $\begin{array}{l}\text { Amount spent by } \\
\text { CLF supporting } \\
\text { Democrat }\end{array}$ & $\begin{array}{l}\text { Federal Election } \\
\text { Commission }\end{array}$ \\
\hline $\begin{array}{l}\text { Dem DCCC } \\
\text { Oppose }\end{array}$ & $\mathrm{T}$ & $\mathrm{F}$ & $\mathrm{F}$ & $\begin{array}{l}\text { Amount spent by } \\
\text { DCCC opposing } \\
\text { Democrat }\end{array}$ & $\begin{array}{l}\text { Federal Election } \\
\text { Commission }\end{array}$ \\
\hline $\begin{array}{l}\text { Dem DCCC } \\
\text { Support }\end{array}$ & $\mathrm{T}$ & $\mathrm{F}$ & $\mathrm{F}$ & $\begin{array}{l}\text { Amount spent by } \\
\text { DCCC supporting } \\
\text { Democrat }\end{array}$ & $\begin{array}{l}\text { Federal Election } \\
\text { Commission }\end{array}$ \\
\hline $\begin{array}{l}\text { Dem Debts Or } \\
\text { Loans Owed By }\end{array}$ & $\mathrm{T}$ & $\mathrm{F}$ & $\mathrm{T}$ & $\begin{array}{l}\text { Democratic debts } \\
\text { or loans owed by } \\
\text { committee }\end{array}$ & $\begin{array}{l}\text { Federal Election } \\
\text { Commission }\end{array}$ \\
\hline $\begin{array}{l}\text { Dem Debts Or } \\
\text { Loans Owed To }\end{array}$ & $\mathrm{T}$ & $\mathrm{F}$ & $\mathrm{T}$ & $\begin{array}{l}\text { Democratic debts } \\
\text { or loans owed to } \\
\text { committee }\end{array}$ & $\begin{array}{l}\text { Federal Election } \\
\text { Commission }\end{array}$ \\
\hline $\begin{array}{l}\text { Dem Ending Cash } \\
\text { On Hand }\end{array}$ & $\mathrm{T}$ & $\mathrm{F}$ & $\mathrm{T}$ & $\begin{array}{l}\text { Democratic ending } \\
\text { cash on hand }\end{array}$ & $\begin{array}{l}\text { Federal Election } \\
\text { Commission }\end{array}$ \\
\hline Dem HMP Oppose & $\mathrm{T}$ & $\mathrm{F}$ & $\mathrm{T}$ & $\begin{array}{l}\text { Amount spent by } \\
\text { HMP opposing } \\
\text { Democrat }\end{array}$ & $\begin{array}{l}\text { Federal Election } \\
\text { Commission }\end{array}$ \\
\hline $\begin{array}{l}\text { Dem HMP } \\
\text { Support }\end{array}$ & $\mathrm{T}$ & $\mathrm{F}$ & $\mathrm{T}$ & $\begin{array}{l}\text { Amount spent by } \\
\text { HMP supporting } \\
\text { Democrat }\end{array}$ & $\begin{array}{l}\text { Federal Election } \\
\text { Commission }\end{array}$ \\
\hline $\begin{array}{l}\text { Dem Ind } \\
\text { Expenditure } \\
\text { Oppose }\end{array}$ & $\mathrm{T}$ & $\mathrm{T}$ & $\mathrm{T}$ & $\begin{array}{l}\text { Independent } \\
\text { Expenditures to } \\
\text { oppose democratic } \\
\text { candidate }\end{array}$ & $\begin{array}{l}\text { Federal Election } \\
\text { Commission }\end{array}$ \\
\hline
\end{tabular}




\begin{tabular}{|c|c|c|c|c|c|}
\hline $\begin{array}{l}\text { Dem Ind } \\
\text { Expenditure } \\
\text { Percent }\end{array}$ & $\mathrm{T}$ & $\mathrm{T}$ & $\mathrm{T}$ & $\begin{array}{l}\text { Democratic } \\
\text { percent of } \\
\text { independent } \\
\text { Expenditures }\end{array}$ & $\begin{array}{l}\text { Federal Election } \\
\text { Commission }\end{array}$ \\
\hline $\begin{array}{l}\text { Dem Ind } \\
\text { Expenditure } \\
\text { Support }\end{array}$ & $\mathrm{T}$ & $\mathrm{T}$ & $\mathrm{T}$ & $\begin{array}{l}\text { Independent } \\
\text { Expenditures to } \\
\text { support democratic } \\
\text { candidate }\end{array}$ & $\begin{array}{l}\text { Federal Election } \\
\text { Commission }\end{array}$ \\
\hline $\begin{array}{l}\text { Dem Individual } \\
\text { Refunds }\end{array}$ & $\mathrm{T}$ & $\mathrm{F}$ & $\mathrm{T}$ & $\begin{array}{l}\text { Democratic } \\
\text { individual refunds }\end{array}$ & $\begin{array}{l}\text { Federal Election } \\
\text { Commission }\end{array}$ \\
\hline $\begin{array}{l}\text { Dem Itemized } \\
\text { Individual } \\
\text { Contributions }\end{array}$ & $\mathrm{T}$ & $\mathrm{F}$ & $\mathrm{T}$ & $\begin{array}{l}\text { Democratic } \\
\text { itemized individual } \\
\text { contributions }\end{array}$ & $\begin{array}{l}\text { Federal Election } \\
\text { Commission }\end{array}$ \\
\hline $\begin{array}{l}\text { Dem Last Vote } \\
\text { Count }\end{array}$ & $\mathrm{T}$ & $\mathrm{F}$ & $\mathrm{F}$ & $\begin{array}{l}\text { Democratic vote } \\
\text { count from } \\
\text { previous cycle } \\
\text { (same cd) }\end{array}$ & $\begin{array}{l}\text { Historical election } \\
\text { results }\end{array}$ \\
\hline $\begin{array}{l}\text { Dem Last Vote } \\
\text { Percent }\end{array}$ & $\mathrm{T}$ & $\mathrm{F}$ & $\mathrm{F}$ & $\begin{array}{l}\text { Democratic vote } \\
\text { percent from } \\
\text { previous cycle } \\
\text { (same cd) }\end{array}$ & $\begin{array}{l}\text { Historical election } \\
\text { results }\end{array}$ \\
\hline $\begin{array}{l}\text { Dem Loans Made } \\
\text { By Candidate }\end{array}$ & $\mathrm{T}$ & $\mathrm{F}$ & $\mathrm{T}$ & $\begin{array}{l}\text { Democratic loans } \\
\text { made by candidate }\end{array}$ & $\begin{array}{l}\text { Federal Election } \\
\text { Commission }\end{array}$ \\
\hline $\begin{array}{l}\text { Dem NAOR } \\
\text { Oppose }\end{array}$ & $\mathrm{T}$ & $\mathrm{T}$ & $\mathrm{T}$ & $\begin{array}{l}\text { Amount spent by } \\
\text { NAOR opposing } \\
\text { Democrat }\end{array}$ & $\begin{array}{l}\text { Federal Election } \\
\text { Commission }\end{array}$ \\
\hline $\begin{array}{l}\text { Dem NAOR } \\
\text { Support }\end{array}$ & $\mathrm{T}$ & $\mathrm{T}$ & $\mathrm{T}$ & $\begin{array}{l}\text { Amount spent by } \\
\text { NAOR supporting } \\
\text { Democrat }\end{array}$ & $\begin{array}{l}\text { Federal Election } \\
\text { Commission }\end{array}$ \\
\hline $\begin{array}{l}\text { Dem NRCC } \\
\text { Oppose }\end{array}$ & $\mathrm{T}$ & $\mathrm{F}$ & $\mathrm{F}$ & $\begin{array}{l}\text { Amount spent by } \\
\text { NRCC opposing } \\
\text { Democrat }\end{array}$ & $\begin{array}{l}\text { Federal Election } \\
\text { Commission }\end{array}$ \\
\hline
\end{tabular}




\begin{tabular}{|c|c|c|c|c|c|}
\hline $\begin{array}{l}\text { Dem NRCC } \\
\text { Support }\end{array}$ & $\mathrm{T}$ & $\mathrm{F}$ & $\mathrm{F}$ & $\begin{array}{l}\text { Amount spent by } \\
\text { NRCC supporting } \\
\text { Democrat }\end{array}$ & $\begin{array}{l}\text { Federal Election } \\
\text { Commission }\end{array}$ \\
\hline $\begin{array}{l}\text { Dem Num } \\
\text { Opponents }\end{array}$ & $\mathrm{T}$ & $\mathrm{T}$ & $\mathrm{F}$ & $\begin{array}{l}\text { Number of } \\
\text { opponents in dem } \\
\text { primary }\end{array}$ & $\begin{array}{l}\text { Historical election } \\
\text { results }\end{array}$ \\
\hline $\begin{array}{l}\text { Dem Offsets To } \\
\text { Operating } \\
\text { Expenditures }\end{array}$ & $\mathrm{T}$ & $\mathrm{F}$ & $\mathrm{T}$ & $\begin{array}{l}\text { Democratic offsets } \\
\text { to operating } \\
\text { Expenditures }\end{array}$ & $\begin{array}{l}\text { Federal Election } \\
\text { Commission }\end{array}$ \\
\hline $\begin{array}{l}\text { Dem Operating } \\
\text { Expenditures }\end{array}$ & $\mathrm{T}$ & $\mathrm{F}$ & $\mathrm{T}$ & $\begin{array}{l}\text { Democratic } \\
\text { operating } \\
\text { Expenditures }\end{array}$ & $\begin{array}{l}\text { Federal Election } \\
\text { Commission }\end{array}$ \\
\hline $\begin{array}{l}\text { Dem Other } \\
\text { Committee } \\
\text { Contributions }\end{array}$ & $\mathrm{T}$ & $\mathrm{F}$ & $\mathrm{T}$ & $\begin{array}{l}\text { Democratic other } \\
\text { committee } \\
\text { contributions }\end{array}$ & $\begin{array}{l}\text { Federal Election } \\
\text { Commission }\end{array}$ \\
\hline $\begin{array}{l}\text { Dem Other } \\
\text { Committee } \\
\text { Refunds }\end{array}$ & $\mathrm{T}$ & $\mathrm{F}$ & $\mathrm{T}$ & $\begin{array}{l}\text { Democratic other } \\
\text { committee refunds }\end{array}$ & $\begin{array}{l}\text { Federal Election } \\
\text { Commission }\end{array}$ \\
\hline $\begin{array}{l}\text { Dem Other } \\
\text { Disbursements }\end{array}$ & $\mathrm{T}$ & $\mathrm{F}$ & $\mathrm{T}$ & $\begin{array}{l}\text { Democratic other } \\
\text { disbursements }\end{array}$ & $\begin{array}{l}\text { Federal Election } \\
\text { Commission }\end{array}$ \\
\hline $\begin{array}{l}\text { Dem Other Loan } \\
\text { Repayments }\end{array}$ & $\mathrm{T}$ & $\mathrm{F}$ & $\mathrm{T}$ & $\begin{array}{l}\text { Democratic other } \\
\text { loan repayments }\end{array}$ & $\begin{array}{l}\text { Federal Election } \\
\text { Commission }\end{array}$ \\
\hline Dem Other Loans & $\mathrm{T}$ & $\mathrm{F}$ & $\mathrm{T}$ & $\begin{array}{l}\text { Democratic other } \\
\text { loans }\end{array}$ & $\begin{array}{l}\text { Federal Election } \\
\text { Commission }\end{array}$ \\
\hline $\begin{array}{l}\text { Dem Other } \\
\text { Receipts }\end{array}$ & $\mathrm{T}$ & $\mathrm{F}$ & $\mathrm{T}$ & $\begin{array}{l}\text { Democratic other } \\
\text { receipts }\end{array}$ & $\begin{array}{l}\text { Federal Election } \\
\text { Commission }\end{array}$ \\
\hline Dem Outspend & $\mathrm{T}$ & $\mathrm{F}$ & $\mathrm{T}$ & $\begin{array}{l}\text { Whether the } \\
\text { democratic } \\
\text { candidate outspent } \\
\text { the republican }\end{array}$ & $\begin{array}{l}\text { Federal Election } \\
\text { Commission }\end{array}$ \\
\hline
\end{tabular}




\begin{tabular}{|c|c|c|c|c|c|}
\hline $\begin{array}{l}\text { Dem Party } \\
\text { Committee } \\
\text { Contributions }\end{array}$ & $\mathrm{T}$ & $\mathrm{F}$ & $\mathrm{T}$ & $\begin{array}{l}\text { Democratic party } \\
\text { committee } \\
\text { contributions }\end{array}$ & $\begin{array}{l}\text { Federal Election } \\
\text { Commission }\end{array}$ \\
\hline $\begin{array}{l}\text { Dem Political } \\
\text { Party Refunds }\end{array}$ & $\mathrm{T}$ & $\mathrm{F}$ & $\mathrm{T}$ & $\begin{array}{l}\text { Democratic Party } \\
\text { refunds }\end{array}$ & $\begin{array}{l}\text { Federal Election } \\
\text { Commission }\end{array}$ \\
\hline $\begin{array}{l}\text { Dem Pres Net } \\
\text { Approve }\end{array}$ & $\mathrm{T}$ & $\mathrm{T}$ & $\mathrm{F}$ & $\begin{array}{l}\text { Net presidential } \\
\text { approval (approval } \\
\text { rating-disapproval } \\
\text { rating) for } \\
\text { Democratic } \\
\text { Presidents, coded } 0 \\
\text { if opposite party } \\
\text { controls } \\
\text { Presidency }\end{array}$ & Gallup \\
\hline Dem Primary HHI & $\mathrm{T}$ & $\mathrm{T}$ & $\mathrm{F}$ & $\begin{array}{l}\text { Herfindahl- } \\
\text { Hirschman index } \\
\text { (HHI) using vote } \\
\text { share distribution } \\
\text { in dem primary }\end{array}$ & $\begin{array}{l}\text { Calculated in- } \\
\text { house }\end{array}$ \\
\hline $\begin{array}{l}\text { Dem Quarterly } \\
\text { Itemized }\end{array}$ & $\mathrm{T}$ & $\mathrm{F}$ & $\mathrm{T}$ & $\begin{array}{l}\text { Democratic } \\
\text { quarterly itemized } \\
\text { contributions }\end{array}$ & $\begin{array}{l}\text { Federal Election } \\
\text { Commission }\end{array}$ \\
\hline $\begin{array}{l}\text { Dem Quarterly } \\
\text { Unitemized }\end{array}$ & $\mathrm{T}$ & $\mathrm{F}$ & $\mathrm{T}$ & $\begin{array}{l}\text { Democratic } \\
\text { quarterly } \\
\text { unitemized } \\
\text { contributions }\end{array}$ & $\begin{array}{l}\text { Federal Election } \\
\text { Commission }\end{array}$ \\
\hline Dem Raised & $\mathrm{T}$ & $\mathrm{F}$ & $\mathrm{T}$ & $\begin{array}{l}\text { Democratic total } \\
\text { raised }\end{array}$ & $\begin{array}{l}\text { Federal Election } \\
\text { Commission }\end{array}$ \\
\hline Dem Spent & $\mathrm{T}$ & $\mathrm{F}$ & $\mathrm{T}$ & $\begin{array}{l}\text { Democratic total } \\
\text { spent }\end{array}$ & $\begin{array}{l}\text { Federal Election } \\
\text { Commission }\end{array}$ \\
\hline
\end{tabular}




\begin{tabular}{|c|c|c|c|c|c|}
\hline $\begin{array}{l}\text { Dem Spent Ind } \\
\text { Support Oppose }\end{array}$ & $\mathrm{T}$ & $\mathrm{T}$ & $\mathrm{T}$ & $\begin{array}{l}\text { Democratic total } \\
\text { spent+Democratic } \\
\text { independent } \\
\text { Expenditures } \\
\text { supporting+Republ } \\
\text { ican independent } \\
\text { Expenditures } \\
\text { opposing }\end{array}$ & $\begin{array}{l}\text { Federal Election } \\
\text { Commission }\end{array}$ \\
\hline $\begin{array}{l}\text { Dem Total } \\
\text { Contribution } \\
\text { Refunds }\end{array}$ & $\mathrm{T}$ & $\mathrm{F}$ & $\mathrm{T}$ & $\begin{array}{l}\text { Democratic total } \\
\text { contributions and } \\
\text { refunds }\end{array}$ & $\begin{array}{l}\text { Federal Election } \\
\text { Commission }\end{array}$ \\
\hline $\begin{array}{l}\text { Dem Total } \\
\text { Contributions }\end{array}$ & $\mathrm{T}$ & $\mathrm{F}$ & $\mathrm{T}$ & $\begin{array}{l}\text { Democratic total } \\
\text { contributions }\end{array}$ & $\begin{array}{l}\text { Federal Election } \\
\text { Commission }\end{array}$ \\
\hline $\begin{array}{l}\text { Dem Total } \\
\text { Individual } \\
\text { Contributions }\end{array}$ & $\mathrm{T}$ & $\mathrm{F}$ & $\mathrm{T}$ & $\begin{array}{l}\text { Democratic total } \\
\text { individual } \\
\text { contributions }\end{array}$ & $\begin{array}{l}\text { Federal Election } \\
\text { Commission }\end{array}$ \\
\hline $\begin{array}{l}\text { Dem Total Loan } \\
\text { Repayments }\end{array}$ & $\mathrm{T}$ & $\mathrm{F}$ & $\mathrm{T}$ & $\begin{array}{l}\text { Democratic total } \\
\text { loan repayments }\end{array}$ & $\begin{array}{l}\text { Federal Election } \\
\text { Commission }\end{array}$ \\
\hline $\begin{array}{l}\text { Dem Total Loans } \\
\text { Received }\end{array}$ & $\mathrm{T}$ & $\mathrm{F}$ & $\mathrm{T}$ & $\begin{array}{l}\text { Democratic total } \\
\text { loans received }\end{array}$ & $\begin{array}{l}\text { Federal Election } \\
\text { Commission }\end{array}$ \\
\hline $\begin{array}{l}\text { Dem Transfers } \\
\text { From Other } \\
\text { Authorized } \\
\text { Committees }\end{array}$ & $\mathrm{T}$ & $\mathrm{F}$ & $\mathrm{T}$ & $\begin{array}{l}\text { Democratic } \\
\text { transfers from } \\
\text { other authorized } \\
\text { committees }\end{array}$ & $\begin{array}{l}\text { Federal Election } \\
\text { Commission }\end{array}$ \\
\hline $\begin{array}{l}\text { Dem Transfers To } \\
\text { Other Authorized } \\
\text { Committees }\end{array}$ & $\mathrm{T}$ & $\mathrm{F}$ & $\mathrm{T}$ & $\begin{array}{l}\text { Democratic } \\
\text { transfers to other } \\
\text { authorized } \\
\text { committees }\end{array}$ & $\begin{array}{l}\text { Federal Election } \\
\text { Commission }\end{array}$ \\
\hline $\begin{array}{l}\text { Dem Unitemized } \\
\text { Individual } \\
\text { Contributions }\end{array}$ & $\mathrm{T}$ & $\mathrm{F}$ & $\mathrm{T}$ & $\begin{array}{l}\text { Democratic } \\
\text { unitemized } \\
\text { individual } \\
\text { contributions }\end{array}$ & $\begin{array}{l}\text { Federal Election } \\
\text { Commission }\end{array}$ \\
\hline
\end{tabular}




\begin{tabular}{|c|c|c|c|c|c|}
\hline $\begin{array}{l}\text { Dem Vote Count } \\
\text { Last3 }\end{array}$ & $\mathrm{T}$ & $\mathrm{T}$ & $\mathrm{F}$ & $\begin{array}{l}\text { Democratic vote } \\
\text { count from } \\
\text { previous } 3 \text { cycles }\end{array}$ & $\begin{array}{l}\text { Historical election } \\
\text { results }\end{array}$ \\
\hline $\begin{array}{l}\text { Dem Vote Percent } \\
\text { Last3 }\end{array}$ & $\mathrm{T}$ & $\mathrm{T}$ & $\mathrm{F}$ & $\begin{array}{l}\text { Democratic vote } \\
\text { percent from } \\
\text { previous } 3 \text { cycles }\end{array}$ & $\begin{array}{l}\text { Historical election } \\
\text { results }\end{array}$ \\
\hline Democrat Gender & $\mathrm{T}$ & $\mathrm{T}$ & $\mathrm{F}$ & $\begin{array}{l}\text { Gender of } \\
\text { Democratic } \\
\text { candidate }\end{array}$ & $\begin{array}{l}\text { Database on } \\
\text { Ideology, Money } \\
\text { in Politics, and } \\
\text { Elections }\end{array}$ \\
\hline $\begin{array}{l}\text { Democrat } \\
\text { Ideology Score }\end{array}$ & $\mathrm{T}$ & $\mathrm{T}$ & $\mathrm{F}$ & $\begin{array}{l}\text { Ideal point } \\
\text { estimate of } \\
\text { Democratic } \\
\text { candidate ideology } \\
\text { based on campaign } \\
\text { finance records } \\
\text { (positive values are } \\
\text { more conservative, } \\
\text { negative values are } \\
\text { more liberal; the } \\
\text { further away from } \\
0 \text { a value is, the } \\
\text { more extreme their } \\
\text { ideology) }\end{array}$ & $\begin{array}{l}\text { Database on } \\
\text { Ideology, Money } \\
\text { in Politics, and } \\
\text { Elections }\end{array}$ \\
\hline $\begin{array}{l}\text { Democratic } \\
\text { candidate } \\
\text { contributions }\end{array}$ & $\mathrm{T}$ & $\mathrm{F}$ & $\mathrm{T}$ & $\begin{array}{l}\text { Democratic } \\
\text { candidate } \\
\text { contributions }\end{array}$ & $\begin{array}{l}\text { Federal Election } \\
\text { Commission }\end{array}$ \\
\hline $\begin{array}{l}\text { Democratic loan } \\
\text { repayments }\end{array}$ & $\mathrm{T}$ & $\mathrm{F}$ & $\mathrm{T}$ & $\begin{array}{l}\text { Democratic loan } \\
\text { repayments }\end{array}$ & $\begin{array}{l}\text { Federal Election } \\
\text { Commission }\end{array}$ \\
\hline $\begin{array}{l}\text { Effective Federal } \\
\text { Funds Rate }\end{array}$ & $\mathrm{F}$ & $\mathrm{F}$ & $\mathrm{T}$ & $\begin{array}{l}\text { Effective Federal } \\
\text { Funds Rate }\end{array}$ & $\begin{array}{l}\text { Federal Reserve } \\
\text { Economic Data }\end{array}$ \\
\hline EV & $\mathrm{F}$ & $\mathrm{F}$ & $\mathrm{T}$ & $\begin{array}{l}\text { Number of } \\
\text { Electoral Votes } \\
\text { available }\end{array}$ & $\mathrm{n} / \mathrm{a}$ \\
\hline
\end{tabular}




\begin{tabular}{|c|c|c|c|c|c|}
\hline $\begin{array}{l}\text { Freshman } \\
\text { Incumbent }\end{array}$ & $\mathrm{T}$ & $\mathrm{F}$ & $\mathrm{F}$ & $\begin{array}{l}0 \text { = not freshman, } \\
1 \text { = freshman } \\
\text { elected previous } \\
\text { general election, } 2 \\
\text { = freshman elected } \\
\text { in special election } \\
\text { more than } 1 \text { year } \\
\text { earlier, } 3 \text { = } \\
\text { freshmen elected } \\
\text { in a special } \\
\text { election during } \\
\text { election year, } 9= \\
\text { seat not defended } \\
\text { by major party } \\
\text { incumbent }\end{array}$ & $\begin{array}{l}\text { Historical election } \\
\text { results }\end{array}$ \\
\hline GDP & $\mathrm{F}$ & $\mathrm{F}$ & $\mathrm{T}$ & $\begin{array}{l}\text { Gross Domestic } \\
\text { Product (GDP) }\end{array}$ & $\begin{array}{l}\text { Federal Reserve } \\
\text { Economic Data }\end{array}$ \\
\hline $\begin{array}{l}\text { Generic Ballot } \\
\text { National } \\
\text { Environment }\end{array}$ & $\mathrm{T}$ & $\mathrm{T}$ & $\mathrm{F}$ & $\begin{array}{l}15 \text { day average of } \\
\text { generic } \\
\text { congressional } \\
\text { ballot of D vs R; } \\
\text { positive values } \\
\text { favor R, negative } \\
\text { values favor D }\end{array}$ & RealClearPolitics \\
\hline Geo Class & $\mathrm{T}$ & $\mathrm{T}$ & $\mathrm{F}$ & $\begin{array}{l}\text { Description of how } \\
\text { rural/urban the } \\
\text { district is (e.g. } \\
\text { "quite_rural", } \\
\text { "extremely_urban" } \\
\text {, } \\
\text { "semi_urban_rural } \\
\text { ") }\end{array}$ & US Census Bureau \\
\hline GNP & $\mathrm{F}$ & $\mathrm{F}$ & $\mathrm{T}$ & $\begin{array}{l}\text { Gross National } \\
\text { Product (GNP) }\end{array}$ & $\begin{array}{l}\text { Federal Reserve } \\
\text { Economic Data }\end{array}$ \\
\hline $\begin{array}{l}\text { GOP Candidate } \\
\text { Contributions }\end{array}$ & $\mathrm{T}$ & $\mathrm{F}$ & $\mathrm{T}$ & $\begin{array}{l}\text { Republican } \\
\text { candidate } \\
\text { contributions }\end{array}$ & $\begin{array}{l}\text { Federal Election } \\
\text { Commission }\end{array}$ \\
\hline
\end{tabular}




\begin{tabular}{|c|c|c|c|c|c|}
\hline $\begin{array}{l}\text { GOP Candidate } \\
\text { Contributions } \\
\text { Score }\end{array}$ & $\mathrm{F}$ & $\mathrm{T}$ & $\mathrm{T}$ & $\begin{array}{l}\text { Difference } \\
\text { between } \\
\text { Republican and } \\
\text { Democratic } \\
\text { candidate } \\
\text { contributions } \\
\text { (larger differences } \\
\text { have values } \\
\text { approaching 1, } \\
\text { smaller differences } \\
\text { have values } \\
\text { approaching 0) }\end{array}$ & $\begin{array}{l}\text { Federal Election } \\
\text { Commission }\end{array}$ \\
\hline $\begin{array}{l}\text { GOP Candidate } \\
\text { Loan Repayments }\end{array}$ & $\mathrm{T}$ & $\mathrm{F}$ & $\mathrm{T}$ & $\begin{array}{l}\text { Republican loan } \\
\text { repayments }\end{array}$ & $\begin{array}{l}\text { Federal Election } \\
\text { Commission }\end{array}$ \\
\hline $\begin{array}{l}\text { GOP Candidate } \\
\text { Loan Repayments } \\
\text { Score }\end{array}$ & $\mathrm{F}$ & $\mathrm{T}$ & $\mathrm{T}$ & $\begin{array}{l}\text { Difference } \\
\text { between } \\
\text { Republican and } \\
\text { Democratic } \\
\text { candidate loan } \\
\text { repayments (larger } \\
\text { differences have } \\
\text { values approaching } \\
\text { 1, smaller } \\
\text { differences have } \\
\text { values approaching } \\
\text { 0) }\end{array}$ & $\begin{array}{l}\text { Federal Election } \\
\text { Commission }\end{array}$ \\
\hline GOP CFG Oppose & $\mathrm{T}$ & $\mathrm{T}$ & $\mathrm{T}$ & $\begin{array}{l}\text { Amount spent by } \\
\text { CFG opposing } \\
\text { Republican }\end{array}$ & $\begin{array}{l}\text { Federal Election } \\
\text { Commission }\end{array}$ \\
\hline GOP CFG Support & $\mathrm{T}$ & $\mathrm{T}$ & $\mathrm{T}$ & $\begin{array}{l}\text { Amount spent by } \\
\text { CFG supporting } \\
\text { Republican }\end{array}$ & $\begin{array}{l}\text { Federal Election } \\
\text { Commission }\end{array}$ \\
\hline GOP CLF Oppose & $\mathrm{T}$ & $\mathrm{F}$ & $\mathrm{T}$ & $\begin{array}{l}\text { Amount spent by } \\
\text { CLF opposing } \\
\text { Republican }\end{array}$ & $\begin{array}{l}\text { Federal Election } \\
\text { Commission }\end{array}$ \\
\hline
\end{tabular}




\begin{tabular}{|c|c|c|c|c|c|}
\hline GOP CLF Support & $\mathrm{T}$ & $\mathrm{F}$ & $\mathrm{T}$ & $\begin{array}{l}\text { Amount spent by } \\
\text { CLF supporting } \\
\text { Republican }\end{array}$ & $\begin{array}{l}\text { Federal Election } \\
\text { Commission }\end{array}$ \\
\hline $\begin{array}{l}\text { GOP DCCC } \\
\text { Oppose }\end{array}$ & $\mathrm{T}$ & $\mathrm{F}$ & $\mathrm{F}$ & $\begin{array}{l}\text { Amount spent by } \\
\text { DCCC opposing } \\
\text { Republican }\end{array}$ & $\begin{array}{l}\text { Federal Election } \\
\text { Commission }\end{array}$ \\
\hline $\begin{array}{l}\text { GOP DCCC } \\
\text { Support }\end{array}$ & $\mathrm{T}$ & $\mathrm{F}$ & $\mathrm{F}$ & $\begin{array}{l}\text { Amount spent by } \\
\text { DCCC supporting } \\
\text { Republican }\end{array}$ & $\begin{array}{l}\text { Federal Election } \\
\text { Commission }\end{array}$ \\
\hline $\begin{array}{l}\text { GOP Debts Or } \\
\text { Loans Owed By }\end{array}$ & $\mathrm{T}$ & $\mathrm{T}$ & $\mathrm{T}$ & $\begin{array}{l}\text { Republican debts } \\
\text { or loans owed by } \\
\text { committee }\end{array}$ & $\begin{array}{l}\text { Federal Election } \\
\text { Commission }\end{array}$ \\
\hline $\begin{array}{l}\text { GOP Debts Or } \\
\text { Loans Owed By } \\
\text { Score }\end{array}$ & $\mathrm{T}$ & $\mathrm{T}$ & $\mathrm{T}$ & $\begin{array}{l}\text { Difference } \\
\text { between } \\
\text { Republican and } \\
\text { Democratic debts } \\
\text { or loans owed by } \\
\text { committee (larger } \\
\text { differences have } \\
\text { values approaching } \\
\text { 1, smaller } \\
\text { differences have } \\
\text { values approaching } \\
\text { 0) }\end{array}$ & $\begin{array}{l}\text { Federal Election } \\
\text { Commission }\end{array}$ \\
\hline $\begin{array}{l}\text { GOP Debts Or } \\
\text { Loans Owed To }\end{array}$ & $\mathrm{T}$ & $\mathrm{F}$ & $\mathrm{T}$ & $\begin{array}{l}\text { Republican debts } \\
\text { or loans owed to } \\
\text { committee }\end{array}$ & $\begin{array}{l}\text { Federal Election } \\
\text { Commission }\end{array}$ \\
\hline
\end{tabular}




\begin{tabular}{|c|c|c|c|c|c|}
\hline $\begin{array}{l}\text { GOP Debts Or } \\
\text { Loans Owed To } \\
\text { Score }\end{array}$ & $\mathrm{F}$ & $\mathrm{T}$ & $\mathrm{T}$ & $\begin{array}{l}\text { Difference } \\
\text { between } \\
\text { Republican and } \\
\text { Democratic debts } \\
\text { or loans owed to } \\
\text { committee (larger } \\
\text { differences have } \\
\text { values approaching } \\
\text { 1, smaller } \\
\text { differences have } \\
\text { values approaching } \\
\text { 0) }\end{array}$ & $\begin{array}{l}\text { Federal Election } \\
\text { Commission }\end{array}$ \\
\hline $\begin{array}{l}\text { GOP Ending Cash } \\
\text { On Hand }\end{array}$ & $\mathrm{T}$ & $\mathrm{F}$ & $\mathrm{T}$ & $\begin{array}{l}\text { Republican ending } \\
\text { cash on hand }\end{array}$ & $\begin{array}{l}\text { Federal Election } \\
\text { Commission }\end{array}$ \\
\hline $\begin{array}{l}\text { GOP Ending Cash } \\
\text { On Hand Score }\end{array}$ & $\mathrm{F}$ & $\mathrm{T}$ & $\mathrm{T}$ & $\begin{array}{l}\text { Difference } \\
\text { between } \\
\text { Republican and } \\
\text { Democratic ending } \\
\text { cash on hand } \\
\text { (larger differences } \\
\text { have values } \\
\text { approaching 1, } \\
\text { smaller differences } \\
\text { have values } \\
\text { approaching 0) }\end{array}$ & $\begin{array}{l}\text { Federal Election } \\
\text { Commission }\end{array}$ \\
\hline GOP HMP Oppose & $\mathrm{T}$ & $\mathrm{F}$ & $\mathrm{T}$ & $\begin{array}{l}\text { Amount spent by } \\
\text { HMP opposing } \\
\text { Republican }\end{array}$ & $\begin{array}{l}\text { Federal Election } \\
\text { Commission }\end{array}$ \\
\hline $\begin{array}{l}\text { GOP HMP } \\
\text { Support }\end{array}$ & $\mathrm{T}$ & $\mathrm{F}$ & $\mathrm{T}$ & $\begin{array}{l}\text { Amount spent by } \\
\text { HMP supporting } \\
\text { Republican }\end{array}$ & $\begin{array}{l}\text { Federal Election } \\
\text { Commission }\end{array}$ \\
\hline $\begin{array}{l}\text { GOP Ind } \\
\text { Expenditure } \\
\text { Oppose }\end{array}$ & $\mathrm{T}$ & $\mathrm{T}$ & $\mathrm{T}$ & $\begin{array}{l}\text { Independent } \\
\text { Expenditures to } \\
\text { oppose republican } \\
\text { candidate }\end{array}$ & $\begin{array}{l}\text { Federal Election } \\
\text { Commission }\end{array}$ \\
\hline
\end{tabular}




\begin{tabular}{|c|c|c|c|c|c|}
\hline $\begin{array}{l}\text { GOP Ind } \\
\text { Expenditure } \\
\text { Percent }\end{array}$ & $\mathrm{T}$ & $\mathrm{T}$ & $\mathrm{T}$ & $\begin{array}{l}\text { Republican percent } \\
\text { of independent } \\
\text { Expenditures }\end{array}$ & $\begin{array}{l}\text { Federal Election } \\
\text { Commission }\end{array}$ \\
\hline $\begin{array}{l}\text { GOP Ind } \\
\text { Expenditure } \\
\text { Support }\end{array}$ & $\mathrm{T}$ & $\mathrm{T}$ & $\mathrm{T}$ & $\begin{array}{l}\text { Independent } \\
\text { Expenditures to } \\
\text { support republican } \\
\text { candidate }\end{array}$ & $\begin{array}{l}\text { Federal Election } \\
\text { Commission }\end{array}$ \\
\hline $\begin{array}{l}\text { GOP Individual } \\
\text { Refunds }\end{array}$ & $\mathrm{T}$ & $\mathrm{F}$ & $\mathrm{T}$ & $\begin{array}{l}\text { Republican } \\
\text { individual refunds }\end{array}$ & $\begin{array}{l}\text { Federal Election } \\
\text { Commission }\end{array}$ \\
\hline $\begin{array}{l}\text { GOP Individual } \\
\text { Refunds Score }\end{array}$ & $\mathrm{F}$ & $\mathrm{T}$ & $\mathrm{T}$ & $\begin{array}{l}\text { Difference } \\
\text { between } \\
\text { Republican and } \\
\text { Democratic } \\
\text { individual refunds } \\
\text { (larger differences } \\
\text { have values } \\
\text { approaching 1, } \\
\text { smaller differences } \\
\text { have values } \\
\text { approaching 0) }\end{array}$ & $\begin{array}{l}\text { Federal Election } \\
\text { Commission }\end{array}$ \\
\hline $\begin{array}{l}\text { GOP Itemized } \\
\text { Individual } \\
\text { Contributions }\end{array}$ & $\mathrm{T}$ & $\mathrm{F}$ & $\mathrm{T}$ & $\begin{array}{l}\text { Republican } \\
\text { itemized individual } \\
\text { contributions }\end{array}$ & $\begin{array}{l}\text { Federal Election } \\
\text { Commission }\end{array}$ \\
\hline $\begin{array}{l}\text { GOP Itemized } \\
\text { Individual } \\
\text { Contributions } \\
\text { Score }\end{array}$ & $\mathrm{F}$ & $\mathrm{T}$ & $\mathrm{T}$ & $\begin{array}{l}\text { Difference } \\
\text { between } \\
\text { Republican and } \\
\text { Democratic } \\
\text { itemized individual } \\
\text { contributions } \\
\text { (larger differences } \\
\text { have values } \\
\text { approaching } 1 \text {, } \\
\text { smaller differences } \\
\text { have values } \\
\text { approaching 0) }\end{array}$ & $\begin{array}{l}\text { Federal Election } \\
\text { Commission }\end{array}$ \\
\hline
\end{tabular}




\begin{tabular}{|c|c|c|c|c|c|}
\hline $\begin{array}{l}\text { GOP Last Vote } \\
\text { Count }\end{array}$ & $\mathrm{T}$ & $\mathrm{F}$ & $\mathrm{F}$ & $\begin{array}{l}\text { GOP vote count } \\
\text { from previous } \\
\text { cycle (same cd) }\end{array}$ & $\begin{array}{l}\text { Historical election } \\
\text { results }\end{array}$ \\
\hline $\begin{array}{l}\text { GOP Last Vote } \\
\text { Percent }\end{array}$ & $\mathrm{T}$ & $\mathrm{F}$ & $\mathrm{F}$ & $\begin{array}{l}\text { GOP vote Percent } \\
\text { from previous } \\
\text { cycle (same cd) }\end{array}$ & $\begin{array}{l}\text { Historical election } \\
\text { results }\end{array}$ \\
\hline $\begin{array}{l}\text { GOP Loans Made } \\
\text { By Candidate }\end{array}$ & $\mathrm{T}$ & $\mathrm{F}$ & $\mathrm{T}$ & $\begin{array}{l}\text { Republican loans } \\
\text { made by candidate }\end{array}$ & $\begin{array}{l}\text { Federal Election } \\
\text { Commission }\end{array}$ \\
\hline $\begin{array}{l}\text { GOP Loans Made } \\
\text { By Candidate } \\
\text { Score }\end{array}$ & $\mathrm{F}$ & $\mathrm{T}$ & $\mathrm{T}$ & $\begin{array}{l}\text { Difference } \\
\text { between } \\
\text { Republican and } \\
\text { Democratic loans } \\
\text { made by candidate } \\
\text { (larger differences } \\
\text { have values } \\
\text { approaching 1, } \\
\text { smaller differences } \\
\text { have values } \\
\text { approaching 0) }\end{array}$ & $\begin{array}{l}\text { Federal Election } \\
\text { Commission }\end{array}$ \\
\hline $\begin{array}{l}\text { GOP NAOR } \\
\text { Oppose }\end{array}$ & $\mathrm{T}$ & $\mathrm{T}$ & $\mathrm{T}$ & $\begin{array}{l}\text { Amount spent by } \\
\text { NAOR opposing } \\
\text { Republican }\end{array}$ & $\begin{array}{l}\text { Federal Election } \\
\text { Commission }\end{array}$ \\
\hline $\begin{array}{l}\text { GOP NAOR } \\
\text { Support }\end{array}$ & $\mathrm{T}$ & $\mathrm{T}$ & $\mathrm{T}$ & $\begin{array}{l}\text { Amount spent by } \\
\text { NAOR supporting } \\
\text { Republican }\end{array}$ & $\begin{array}{l}\text { Federal Election } \\
\text { Commission }\end{array}$ \\
\hline $\begin{array}{l}\text { GOP NRCC } \\
\text { Oppose }\end{array}$ & $\mathrm{T}$ & $\mathrm{F}$ & $\mathrm{F}$ & $\begin{array}{l}\text { Amount spent by } \\
\text { NRCC opposing } \\
\text { Republican }\end{array}$ & $\begin{array}{l}\text { Federal Election } \\
\text { Commission }\end{array}$ \\
\hline $\begin{array}{l}\text { GOP NRCC } \\
\text { Support }\end{array}$ & $\mathrm{T}$ & $\mathrm{F}$ & $\mathrm{F}$ & $\begin{array}{l}\text { Amount spent by } \\
\text { NRCC supporting } \\
\text { Republican }\end{array}$ & $\begin{array}{l}\text { Federal Election } \\
\text { Commission }\end{array}$ \\
\hline $\begin{array}{l}\text { GOP Num } \\
\text { Opponents }\end{array}$ & $\mathrm{T}$ & $\mathrm{T}$ & $\mathrm{F}$ & $\begin{array}{l}\text { Number of } \\
\text { opponents in GOP } \\
\text { primary }\end{array}$ & $\begin{array}{l}\text { Historical election } \\
\text { results }\end{array}$ \\
\hline
\end{tabular}




\begin{tabular}{|c|c|c|c|c|c|}
\hline $\begin{array}{l}\text { GOP Offsets To } \\
\text { Operating } \\
\text { Expenditures }\end{array}$ & $\mathrm{T}$ & $\mathrm{F}$ & $\mathrm{T}$ & $\begin{array}{l}\text { Republican offsets } \\
\text { to operating } \\
\text { Expenditures }\end{array}$ & $\begin{array}{l}\text { Federal Election } \\
\text { Commission }\end{array}$ \\
\hline $\begin{array}{l}\text { GOP Offsets To } \\
\text { Operating } \\
\text { Expenditures } \\
\text { Score }\end{array}$ & $\mathrm{F}$ & $\mathrm{T}$ & $\mathrm{T}$ & $\begin{array}{l}\text { Difference } \\
\text { between } \\
\text { Republican and } \\
\text { Democratic offsets } \\
\text { to operating } \\
\text { Expenditures } \\
\text { (larger differences } \\
\text { have values } \\
\text { approaching 1, } \\
\text { smaller differences } \\
\text { have values } \\
\text { approaching 0) }\end{array}$ & $\begin{array}{l}\text { Federal Election } \\
\text { Commission }\end{array}$ \\
\hline $\begin{array}{l}\text { GOP Operating } \\
\text { Expenditures }\end{array}$ & $\mathrm{T}$ & $\mathrm{F}$ & $\mathrm{T}$ & $\begin{array}{l}\text { Republican } \\
\text { operating } \\
\text { Expenditures }\end{array}$ & $\begin{array}{l}\text { Federal Election } \\
\text { Commission }\end{array}$ \\
\hline $\begin{array}{l}\text { GOP Operating } \\
\text { Expenditures } \\
\text { Score }\end{array}$ & $\mathrm{F}$ & $\mathrm{T}$ & $\mathrm{T}$ & $\begin{array}{l}\text { Difference } \\
\text { between } \\
\text { Republican and } \\
\text { Democratic } \\
\text { operating } \\
\text { Expenditures } \\
\text { (larger differences } \\
\text { have values } \\
\text { approaching 1, } \\
\text { smaller differences } \\
\text { have values } \\
\text { approaching 0) }\end{array}$ & $\begin{array}{l}\text { Federal Election } \\
\text { Commission }\end{array}$ \\
\hline $\begin{array}{l}\text { GOP Other } \\
\text { Committee } \\
\text { Contributions }\end{array}$ & $\mathrm{T}$ & $\mathrm{F}$ & $\mathrm{T}$ & $\begin{array}{l}\text { Republican other } \\
\text { committee } \\
\text { contributions }\end{array}$ & $\begin{array}{l}\text { Federal Election } \\
\text { Commission }\end{array}$ \\
\hline
\end{tabular}




\begin{tabular}{|c|c|c|c|c|c|}
\hline $\begin{array}{l}\text { GOP Other } \\
\text { Committee } \\
\text { Contributions } \\
\text { Score }\end{array}$ & $\mathrm{F}$ & $\mathrm{T}$ & $\mathrm{T}$ & $\begin{array}{l}\text { Difference } \\
\text { between } \\
\text { Republican and } \\
\text { Democratic other } \\
\text { committee } \\
\text { contributions } \\
\text { (larger differences } \\
\text { have values } \\
\text { approaching 1, } \\
\text { smaller differences } \\
\text { have values } \\
\text { approaching 0) }\end{array}$ & $\begin{array}{l}\text { Federal Election } \\
\text { Commission }\end{array}$ \\
\hline $\begin{array}{l}\text { GOP Other } \\
\text { Committee } \\
\text { Refunds }\end{array}$ & $\mathrm{T}$ & $\mathrm{F}$ & $\mathrm{T}$ & $\begin{array}{l}\text { Republican other } \\
\text { committee refunds }\end{array}$ & $\begin{array}{l}\text { Federal Election } \\
\text { Commission }\end{array}$ \\
\hline $\begin{array}{l}\text { GOP Other } \\
\text { Committee } \\
\text { Refunds Score }\end{array}$ & $\mathrm{F}$ & $\mathrm{T}$ & $\mathrm{T}$ & $\begin{array}{l}\text { Difference } \\
\text { between } \\
\text { Republican and } \\
\text { Democratic other } \\
\text { committee refunds } \\
\text { (larger differences } \\
\text { have values } \\
\text { approaching 1, } \\
\text { smaller differences } \\
\text { have values } \\
\text { approaching 0) }\end{array}$ & $\begin{array}{l}\text { Federal Election } \\
\text { Commission }\end{array}$ \\
\hline $\begin{array}{l}\text { GOP Other } \\
\text { Disbursements }\end{array}$ & $\mathrm{T}$ & $\mathrm{F}$ & $\mathrm{T}$ & $\begin{array}{l}\text { Republican other } \\
\text { disbursements }\end{array}$ & $\begin{array}{l}\text { Federal Election } \\
\text { Commission }\end{array}$ \\
\hline
\end{tabular}




\begin{tabular}{|c|c|c|c|c|c|}
\hline $\begin{array}{l}\text { GOP Other } \\
\text { Disbursements } \\
\text { Score }\end{array}$ & $\mathrm{F}$ & $\mathrm{T}$ & $\mathrm{T}$ & $\begin{array}{l}\text { Difference } \\
\text { between } \\
\text { Republican and } \\
\text { Democratic other } \\
\text { disbursements } \\
\text { (larger differences } \\
\text { have values } \\
\text { approaching 1, } \\
\text { smaller differences } \\
\text { have values } \\
\text { approaching } 0 \text { ) }\end{array}$ & $\begin{array}{l}\text { Federal Election } \\
\text { Commission }\end{array}$ \\
\hline $\begin{array}{l}\text { GOP Other Loan } \\
\text { Repayments }\end{array}$ & $\mathrm{T}$ & $\mathrm{F}$ & $\mathrm{T}$ & $\begin{array}{l}\text { Republican other } \\
\text { loan repayments }\end{array}$ & $\begin{array}{l}\text { Federal Election } \\
\text { Commission }\end{array}$ \\
\hline $\begin{array}{l}\text { GOP Other Loan } \\
\text { Repayments Score }\end{array}$ & $\mathrm{F}$ & $\mathrm{T}$ & $\mathrm{T}$ & $\begin{array}{l}\text { Difference } \\
\text { between } \\
\text { Republican and } \\
\text { Democratic other } \\
\text { loan repayments } \\
\text { (larger differences } \\
\text { have values } \\
\text { approaching 1, } \\
\text { smaller differences } \\
\text { have values } \\
\text { approaching } 0 \text { ) }\end{array}$ & $\begin{array}{l}\text { Federal Election } \\
\text { Commission }\end{array}$ \\
\hline GOP Other Loans & $\mathrm{T}$ & F & $\mathrm{T}$ & $\begin{array}{l}\text { Republican other } \\
\text { loans }\end{array}$ & $\begin{array}{l}\text { Federal Election } \\
\text { Commission }\end{array}$ \\
\hline $\begin{array}{l}\text { GOP Other Loans } \\
\text { Score }\end{array}$ & $\mathrm{F}$ & $\mathrm{T}$ & $\mathrm{T}$ & $\begin{array}{l}\text { Difference } \\
\text { between } \\
\text { Republican and } \\
\text { Democratic other } \\
\text { loans (larger } \\
\text { differences have } \\
\text { values approaching } \\
1 \text {, smaller } \\
\text { differences have } \\
\text { values approaching } \\
0 \text { ) }\end{array}$ & $\begin{array}{l}\text { Federal Election } \\
\text { Commission }\end{array}$ \\
\hline
\end{tabular}




\begin{tabular}{|c|c|c|c|c|c|}
\hline $\begin{array}{l}\text { GOP Other } \\
\text { Receipts }\end{array}$ & $\mathrm{T}$ & $\mathrm{F}$ & $\mathrm{T}$ & $\begin{array}{l}\text { Republican other } \\
\text { receipts }\end{array}$ & $\begin{array}{l}\text { Federal Election } \\
\text { Commission }\end{array}$ \\
\hline $\begin{array}{l}\text { GOP Other } \\
\text { Receipts Score }\end{array}$ & $\mathrm{F}$ & $\mathrm{T}$ & $\mathrm{T}$ & $\begin{array}{l}\text { Difference } \\
\text { between } \\
\text { Republican and } \\
\text { Democratic other } \\
\text { receipts (larger } \\
\text { differences have } \\
\text { values approaching } \\
\text { 1, smaller } \\
\text { differences have } \\
\text { values approaching } \\
\text { 0) }\end{array}$ & $\begin{array}{l}\text { Federal Election } \\
\text { Commission }\end{array}$ \\
\hline $\begin{array}{l}\text { GOP Party } \\
\text { Committee } \\
\text { Contributions }\end{array}$ & $\mathrm{T}$ & $\mathrm{F}$ & $\mathrm{T}$ & $\begin{array}{l}\text { Republican party } \\
\text { committee } \\
\text { contributions }\end{array}$ & $\begin{array}{l}\text { Federal Election } \\
\text { Commission }\end{array}$ \\
\hline $\begin{array}{l}\text { GOP Party } \\
\text { Committee } \\
\text { Contributions } \\
\text { Score }\end{array}$ & $\mathrm{F}$ & $\mathrm{T}$ & $\mathrm{T}$ & $\begin{array}{l}\text { Difference } \\
\text { between } \\
\text { Republican and } \\
\text { Democratic party } \\
\text { committee } \\
\text { contributions } \\
\text { (larger differences } \\
\text { have values } \\
\text { approaching 1, } \\
\text { smaller differences } \\
\text { have values } \\
\text { approaching 0) }\end{array}$ & $\begin{array}{l}\text { Federal Election } \\
\text { Commission }\end{array}$ \\
\hline $\begin{array}{l}\text { GOP Political } \\
\text { Party Refunds }\end{array}$ & $\mathrm{T}$ & $\mathrm{F}$ & $\mathrm{T}$ & $\begin{array}{l}\text { Republican Party } \\
\text { refunds }\end{array}$ & $\begin{array}{l}\text { Federal Election } \\
\text { Commission }\end{array}$ \\
\hline
\end{tabular}




\begin{tabular}{|c|c|c|c|c|c|}
\hline $\begin{array}{l}\text { GOP Political } \\
\text { Party Refunds } \\
\text { Score }\end{array}$ & $\mathrm{F}$ & $\mathrm{T}$ & $\mathrm{T}$ & $\begin{array}{l}\text { Difference } \\
\text { between } \\
\text { Republican and } \\
\text { Democratic party } \\
\text { refunds (larger } \\
\text { differences have } \\
\text { values approaching } \\
\text { 1, smaller } \\
\text { differences have } \\
\text { values approaching } \\
\text { 0) }\end{array}$ & $\begin{array}{l}\text { Federal Election } \\
\text { Commission }\end{array}$ \\
\hline $\begin{array}{l}\text { GOP Pres Net } \\
\text { Approve }\end{array}$ & $\mathrm{T}$ & $\mathrm{T}$ & $\mathrm{F}$ & $\begin{array}{l}\text { Net presidential } \\
\text { approval (approval } \\
\text { rating-disapproval } \\
\text { rating) for } \\
\text { Republican } \\
\text { Presidents, coded } 0 \\
\text { if opposite party } \\
\text { controls } \\
\text { Presidency }\end{array}$ & Gallup \\
\hline GOP Primary HHI & $\mathrm{T}$ & $\mathrm{T}$ & $\mathrm{F}$ & $\begin{array}{l}\text { HHI using vote } \\
\text { share distribution } \\
\text { in GOP primary }\end{array}$ & $\begin{array}{l}\text { Calculated in- } \\
\text { house }\end{array}$ \\
\hline $\begin{array}{l}\text { GOP Quarterly } \\
\text { Itemized }\end{array}$ & $\mathrm{T}$ & $\mathrm{F}$ & $\mathrm{T}$ & $\begin{array}{l}\text { Republican } \\
\text { quarterly itemized } \\
\text { contributions }\end{array}$ & $\begin{array}{l}\text { Federal Election } \\
\text { Commission }\end{array}$ \\
\hline $\begin{array}{l}\text { GOP Quarterly } \\
\text { Itemized Score }\end{array}$ & $\mathrm{F}$ & $\mathrm{T}$ & $\mathrm{T}$ & $\begin{array}{l}\text { Difference } \\
\text { between } \\
\text { Republican and } \\
\text { Democratic } \\
\text { quarterly itemized } \\
\text { contributions } \\
\text { (larger differences } \\
\text { have values } \\
\text { approaching 1, } \\
\text { smaller differences } \\
\text { have values } \\
\text { approaching 0) }\end{array}$ & $\begin{array}{l}\text { Federal Election } \\
\text { Commission }\end{array}$ \\
\hline
\end{tabular}




\begin{tabular}{|c|c|c|c|c|c|}
\hline $\begin{array}{l}\text { GOP Quarterly } \\
\text { Unitemized }\end{array}$ & $\mathrm{T}$ & $\mathrm{F}$ & $\mathrm{T}$ & $\begin{array}{l}\text { Republican } \\
\text { quarterly } \\
\text { unitemized } \\
\text { contributions }\end{array}$ & $\begin{array}{l}\text { Federal Election } \\
\text { Commission }\end{array}$ \\
\hline $\begin{array}{l}\text { GOP Quarterly } \\
\text { Unitemized Score }\end{array}$ & $\mathrm{F}$ & $\mathrm{T}$ & $\mathrm{T}$ & $\begin{array}{l}\text { Difference } \\
\text { between } \\
\text { Republican and } \\
\text { Democratic } \\
\text { quarterly } \\
\text { unitemized } \\
\text { contributions } \\
\text { (larger differences } \\
\text { have values } \\
\text { approaching 1, } \\
\text { smaller differences } \\
\text { have values } \\
\text { approaching 0) }\end{array}$ & $\begin{array}{l}\text { Federal Election } \\
\text { Commission }\end{array}$ \\
\hline GOP Raised & $\mathrm{T}$ & $\mathrm{F}$ & $\mathrm{T}$ & $\begin{array}{l}\text { Republican total } \\
\text { raised }\end{array}$ & $\begin{array}{l}\text { Federal Election } \\
\text { Commission }\end{array}$ \\
\hline GOP Raised Score & $\mathrm{T}$ & $\mathrm{T}$ & $\mathrm{T}$ & $\begin{array}{l}\text { Difference } \\
\text { between } \\
\text { Republican and } \\
\text { Democratic total } \\
\text { raised (larger } \\
\text { differences have } \\
\text { values approaching } \\
\text { 1, smaller } \\
\text { differences have } \\
\text { values approaching } \\
\text { 0) }\end{array}$ & $\begin{array}{l}\text { Federal Election } \\
\text { Commission }\end{array}$ \\
\hline GOP Spent & $\mathrm{T}$ & $\mathrm{F}$ & $\mathrm{T}$ & $\begin{array}{l}\text { Republican total } \\
\text { spent }\end{array}$ & $\begin{array}{l}\text { Federal Election } \\
\text { Commission }\end{array}$ \\
\hline
\end{tabular}




\begin{tabular}{|c|c|c|c|c|c|}
\hline $\begin{array}{l}\text { GOP Spent Ind } \\
\text { Support Oppose }\end{array}$ & $\mathrm{T}$ & $\mathrm{T}$ & $\mathrm{T}$ & $\begin{array}{l}\text { Republican total } \\
\text { spent+Republican } \\
\text { independent } \\
\text { Expenditures } \\
\text { supporting+Democ } \\
\text { ratic independent } \\
\text { Expenditures } \\
\text { opposing }\end{array}$ & $\begin{array}{l}\text { Federal Election } \\
\text { Commission }\end{array}$ \\
\hline GOP Spent Score & $\mathrm{T}$ & $\mathrm{T}$ & $\mathrm{T}$ & $\begin{array}{l}\text { Difference } \\
\text { between } \\
\text { Republican and } \\
\text { Democratic total } \\
\text { spent (larger } \\
\text { differences have } \\
\text { values approaching } \\
\text { 1, smaller } \\
\text { differences have } \\
\text { values approaching } \\
\text { 0) }\end{array}$ & $\begin{array}{l}\text { Federal Election } \\
\text { Commission }\end{array}$ \\
\hline $\begin{array}{l}\text { GOP Total } \\
\text { Contribution } \\
\text { Refunds }\end{array}$ & $\mathrm{T}$ & $\mathrm{F}$ & $\mathrm{T}$ & $\begin{array}{l}\text { Republican total } \\
\text { contributions and } \\
\text { refunds }\end{array}$ & $\begin{array}{l}\text { Federal Election } \\
\text { Commission }\end{array}$ \\
\hline $\begin{array}{l}\text { GOP Total } \\
\text { Contribution } \\
\text { Refunds Score }\end{array}$ & $\mathrm{F}$ & $\mathrm{T}$ & $\mathrm{T}$ & $\begin{array}{l}\text { Difference } \\
\text { between } \\
\text { Republican and } \\
\text { Democratic total } \\
\text { contributions and } \\
\text { refunds (larger } \\
\text { differences have } \\
\text { values approaching } \\
\text { 1, smaller } \\
\text { differences have } \\
\text { values approaching } \\
\text { 0) }\end{array}$ & $\begin{array}{l}\text { Federal Election } \\
\text { Commission }\end{array}$ \\
\hline $\begin{array}{l}\text { GOP Total } \\
\text { Contributions }\end{array}$ & $\mathrm{T}$ & $\mathrm{F}$ & $\mathrm{T}$ & $\begin{array}{l}\text { Republican total } \\
\text { contributions }\end{array}$ & $\begin{array}{l}\text { Federal Election } \\
\text { Commission }\end{array}$ \\
\hline
\end{tabular}




\begin{tabular}{|c|c|c|c|c|c|}
\hline $\begin{array}{l}\text { GOP Total } \\
\text { Contributions } \\
\text { Score }\end{array}$ & $\mathrm{F}$ & $\mathrm{T}$ & $\mathrm{T}$ & $\begin{array}{l}\text { Difference } \\
\text { between } \\
\text { Republican and } \\
\text { Democratic total } \\
\text { contributions } \\
\text { (larger differences } \\
\text { have values } \\
\text { approaching 1, } \\
\text { smaller differences } \\
\text { have values } \\
\text { approaching 0) }\end{array}$ & $\begin{array}{l}\text { Federal Election } \\
\text { Commission }\end{array}$ \\
\hline $\begin{array}{l}\text { GOP Total } \\
\text { Individual } \\
\text { Contributions }\end{array}$ & $\mathrm{T}$ & $\mathrm{F}$ & $\mathrm{T}$ & $\begin{array}{l}\text { Republican total } \\
\text { individual } \\
\text { contributions }\end{array}$ & $\begin{array}{l}\text { Federal Election } \\
\text { Commission }\end{array}$ \\
\hline $\begin{array}{l}\text { GOP Total } \\
\text { Individual } \\
\text { Contributions } \\
\text { Score }\end{array}$ & $\mathrm{F}$ & $\mathrm{T}$ & $\mathrm{T}$ & $\begin{array}{l}\text { Difference } \\
\text { between } \\
\text { Republican and } \\
\text { Democratic total } \\
\text { individual } \\
\text { contributions } \\
\text { (larger differences } \\
\text { have values } \\
\text { approaching 1, } \\
\text { smaller differences } \\
\text { have values } \\
\text { approaching 0) }\end{array}$ & $\begin{array}{l}\text { Federal Election } \\
\text { Commission }\end{array}$ \\
\hline $\begin{array}{l}\text { GOP Total Loan } \\
\text { Repayments }\end{array}$ & $\mathrm{T}$ & $\mathrm{F}$ & $\mathrm{T}$ & $\begin{array}{l}\text { Republican total } \\
\text { loan repayments }\end{array}$ & $\begin{array}{l}\text { Federal Election } \\
\text { Commission }\end{array}$ \\
\hline
\end{tabular}




\begin{tabular}{|c|c|c|c|c|c|}
\hline $\begin{array}{l}\text { GOP Total Loan } \\
\text { Repayments Score }\end{array}$ & $\mathrm{F}$ & $\mathrm{T}$ & $\mathrm{T}$ & $\begin{array}{l}\text { Difference } \\
\text { between } \\
\text { Republican and } \\
\text { Democratic total } \\
\text { loan repayments } \\
\text { (larger differences } \\
\text { have values } \\
\text { approaching 1, } \\
\text { smaller differences } \\
\text { have values } \\
\text { approaching 0) }\end{array}$ & $\begin{array}{l}\text { Federal Election } \\
\text { Commission }\end{array}$ \\
\hline $\begin{array}{l}\text { GOP Total Loans } \\
\text { Received }\end{array}$ & $\mathrm{T}$ & $\mathrm{F}$ & $\mathrm{T}$ & $\begin{array}{l}\text { Republican total } \\
\text { loans received }\end{array}$ & $\begin{array}{l}\text { Federal Election } \\
\text { Commission }\end{array}$ \\
\hline $\begin{array}{l}\text { GOP Total Loans } \\
\text { Received Score }\end{array}$ & $\mathrm{F}$ & $\mathrm{T}$ & $\mathrm{T}$ & $\begin{array}{l}\text { Difference } \\
\text { between } \\
\text { Republican and } \\
\text { Democratic total } \\
\text { loans received } \\
\text { (larger differences } \\
\text { have values } \\
\text { approaching 1, } \\
\text { smaller differences } \\
\text { have values } \\
\text { approaching 0) }\end{array}$ & $\begin{array}{l}\text { Federal Election } \\
\text { Commission }\end{array}$ \\
\hline $\begin{array}{l}\text { GOP Transfers } \\
\text { From Other } \\
\text { Authorized } \\
\text { Committees }\end{array}$ & $\mathrm{T}$ & $\mathrm{F}$ & $\mathrm{T}$ & $\begin{array}{l}\text { Republican } \\
\text { transfers from } \\
\text { other authorized } \\
\text { committees }\end{array}$ & $\begin{array}{l}\text { Federal Election } \\
\text { Commission }\end{array}$ \\
\hline
\end{tabular}




\begin{tabular}{|c|c|c|c|c|c|}
\hline $\begin{array}{l}\text { GOP Transfers } \\
\text { From Other } \\
\text { Authorized } \\
\text { Committees Score }\end{array}$ & $\mathrm{F}$ & $\mathrm{T}$ & $\mathrm{T}$ & $\begin{array}{l}\text { Difference } \\
\text { between } \\
\text { Republican and } \\
\text { Democratic } \\
\text { transfers from } \\
\text { other authorized } \\
\text { committees (larger } \\
\text { differences have } \\
\text { values approaching } \\
\text { 1, smaller } \\
\text { differences have } \\
\text { values approaching } \\
\text { 0) }\end{array}$ & $\begin{array}{l}\text { Federal Election } \\
\text { Commission }\end{array}$ \\
\hline $\begin{array}{l}\text { GOP Transfers To } \\
\text { Other Authorized } \\
\text { Committees }\end{array}$ & $\mathrm{T}$ & $\mathrm{F}$ & $\mathrm{T}$ & $\begin{array}{l}\text { Republican } \\
\text { transfers to other } \\
\text { authorized } \\
\text { committees }\end{array}$ & $\begin{array}{l}\text { Federal Election } \\
\text { Commission }\end{array}$ \\
\hline $\begin{array}{l}\text { GOP Transfers To } \\
\text { Other Authorized } \\
\text { Committees Score }\end{array}$ & $\mathrm{F}$ & $\mathrm{T}$ & $\mathrm{T}$ & $\begin{array}{l}\text { Difference } \\
\text { between } \\
\text { Republican and } \\
\text { Democratic } \\
\text { transfers to other } \\
\text { authorized } \\
\text { committees (larger } \\
\text { differences have } \\
\text { values approaching } \\
\text { 1, smaller } \\
\text { differences have } \\
\text { values approaching } \\
\text { 0) }\end{array}$ & $\begin{array}{l}\text { Federal Election } \\
\text { Commission }\end{array}$ \\
\hline $\begin{array}{l}\text { GOP Unitemized } \\
\text { Individual } \\
\text { Contributions }\end{array}$ & $\mathrm{T}$ & $\mathrm{F}$ & $\mathrm{T}$ & $\begin{array}{l}\text { Republican } \\
\text { unitemized } \\
\text { individual } \\
\text { contributions }\end{array}$ & $\begin{array}{l}\text { Federal Election } \\
\text { Commission }\end{array}$ \\
\hline
\end{tabular}




\begin{tabular}{|c|c|c|c|c|c|}
\hline $\begin{array}{l}\text { GOP Unitemized } \\
\text { Individual } \\
\text { Contributions } \\
\text { Score }\end{array}$ & $\mathrm{F}$ & $\mathrm{T}$ & $\mathrm{T}$ & $\begin{array}{l}\text { Difference } \\
\text { between } \\
\text { Republican and } \\
\text { Democratic } \\
\text { unitemized } \\
\text { individual } \\
\text { contributions } \\
\text { (larger differences } \\
\text { have values } \\
\text { approaching 1, } \\
\text { smaller differences } \\
\text { have values } \\
\text { approaching 0) }\end{array}$ & $\begin{array}{l}\text { Federal Election } \\
\text { Commission }\end{array}$ \\
\hline $\begin{array}{l}\text { GOP Vote Count } \\
\text { Last3 }\end{array}$ & $\mathrm{T}$ & $\mathrm{T}$ & $\mathrm{F}$ & $\begin{array}{l}\text { Republican vote } \\
\text { count from } \\
\text { previous } 3 \text { cycles }\end{array}$ & $\begin{array}{l}\text { Historical election } \\
\text { results }\end{array}$ \\
\hline $\begin{array}{l}\text { GOP Vote Percent } \\
\text { Last3 }\end{array}$ & $\mathrm{T}$ & $\mathrm{T}$ & $\mathrm{F}$ & $\begin{array}{l}\text { Republican vote } \\
\text { percent from } \\
\text { previous } 3 \text { cycles }\end{array}$ & $\begin{array}{l}\text { Historical election } \\
\text { results }\end{array}$ \\
\hline GOP Win & $\mathrm{T}$ & $\mathrm{T}$ & $\mathrm{F}$ & $\begin{array}{l}\text { Response variable, } \\
\text { boolean indicating } \\
\text { if republican won }\end{array}$ & $\begin{array}{l}\text { Historical election } \\
\text { results }\end{array}$ \\
\hline $\begin{array}{l}\text { Grn Last Vote } \\
\text { Percent }\end{array}$ & $\mathrm{T}$ & $\mathrm{F}$ & $\mathrm{F}$ & $\begin{array}{l}\text { Green party vote } \\
\text { Percent from } \\
\text { previous cycle } \\
\text { (same cd) }\end{array}$ & $\begin{array}{l}\text { Historical election } \\
\text { results }\end{array}$ \\
\hline Hispanic Pct & $\mathrm{F}$ & $\mathrm{F}$ & $\mathrm{T}$ & $\begin{array}{l}\text { Hispanic } \\
\text { population percent }\end{array}$ & US Census Bureau \\
\hline HMP Involved & $\mathrm{T}$ & $\mathrm{F}$ & $\mathrm{T}$ & $\begin{array}{l}\text { HMP spent money } \\
\mathrm{T} / \mathrm{F}\end{array}$ & $\begin{array}{l}\text { Federal Election } \\
\text { Commission }\end{array}$ \\
\hline HMP Percent & $\mathrm{T}$ & $\mathrm{F}$ & $\mathrm{T}$ & $\begin{array}{l}\text { Percent of } \\
\text { spending from } \\
\text { HMP }\end{array}$ & $\begin{array}{l}\text { Federal Election } \\
\text { Commission }\end{array}$ \\
\hline
\end{tabular}




\begin{tabular}{|c|c|c|c|c|c|}
\hline Incumbent & $\mathrm{T}$ & $\mathrm{T}$ & $\mathrm{F}$ & $\begin{array}{l}1 \text { = GOP } \\
\text { incumbent, } 0 \text { = no } \\
\text { incumbent, }-1 \text { = } \\
\text { Dem incumbent }\end{array}$ & $\begin{array}{l}\text { Historical election } \\
\text { results }\end{array}$ \\
\hline $\begin{array}{l}\text { Ind Last Vote } \\
\text { Percent }\end{array}$ & $\mathrm{T}$ & $\mathrm{F}$ & $\mathrm{F}$ & $\begin{array}{l}\text { Independent party } \\
\text { vote Percent from } \\
\text { previous cycle } \\
\text { (same cd) }\end{array}$ & $\begin{array}{l}\text { Historical election } \\
\text { results }\end{array}$ \\
\hline $\begin{array}{l}\text { Index of } \\
\text { Consumer } \\
\text { Sentiment }\end{array}$ & $\mathrm{F}$ & $\mathrm{F}$ & $\mathrm{T}$ & $\begin{array}{l}\text { Index of Consumer } \\
\text { Sentiment }\end{array}$ & $\begin{array}{l}\text { Federal Reserve } \\
\text { Economic Data }\end{array}$ \\
\hline $\begin{array}{l}\text { Industrial } \\
\text { Production Index }\end{array}$ & $\mathrm{F}$ & $\mathrm{F}$ & $\mathrm{T}$ & $\begin{array}{l}\text { Industrial } \\
\text { Production Index }\end{array}$ & $\begin{array}{l}\text { Federal Reserve } \\
\text { Economic Data }\end{array}$ \\
\hline Last D 2 Party Pct & $\mathrm{F}$ & $\mathrm{F}$ & $\mathrm{T}$ & $\begin{array}{l}\text { Democratic } \\
\text { percentage of two- } \\
\text { party vote in } \\
\text { previous election }\end{array}$ & $\begin{array}{l}\text { Historical election } \\
\text { results }\end{array}$ \\
\hline Last D Overall Pct & $\mathrm{F}$ & $\mathrm{F}$ & $\mathrm{T}$ & $\begin{array}{l}\text { Democratic } \\
\text { percentage of } \\
\text { overall vote in } \\
\text { previous election }\end{array}$ & $\begin{array}{l}\text { Historical election } \\
\text { results }\end{array}$ \\
\hline $\begin{array}{l}\text { Last D Pres } \\
\text { Percent }\end{array}$ & $\mathrm{T}$ & $\mathrm{F}$ & $\mathrm{F}$ & $\begin{array}{l}\text { Democratlu2019s } \\
\text { share of two-party } \\
\text { vote, previous } \\
\text { election }\end{array}$ & $\begin{array}{l}\text { Historical election } \\
\text { results }\end{array}$ \\
\hline Last GOP Percent & $\mathrm{F}$ & $\mathrm{T}$ & $\mathrm{F}$ & $\begin{array}{l}\text { Same as "GOP } \\
\text { Last Vote Percent" } \\
\text { variable, but for } \\
\text { the Senate }\end{array}$ & $\begin{array}{l}\text { Historical election } \\
\text { results }\end{array}$ \\
\hline Last R 2 Party Pct & $\mathrm{F}$ & $\mathrm{F}$ & $\mathrm{T}$ & $\begin{array}{l}\text { Republican } \\
\text { percentage of two- } \\
\text { party vote in } \\
\text { previous election }\end{array}$ & $\begin{array}{l}\text { Historical election } \\
\text { results }\end{array}$ \\
\hline
\end{tabular}




\begin{tabular}{|c|c|c|c|c|c|}
\hline Last R Overall Pct & $\mathrm{F}$ & $\mathrm{F}$ & $\mathrm{T}$ & $\begin{array}{l}\text { Republican } \\
\text { percentage of } \\
\text { overall vote in } \\
\text { previous election }\end{array}$ & $\begin{array}{l}\text { Historical election } \\
\text { results }\end{array}$ \\
\hline $\begin{array}{l}\text { Lib Last Vote } \\
\text { Percent }\end{array}$ & $\mathrm{T}$ & $\mathrm{F}$ & $\mathrm{F}$ & $\begin{array}{l}\text { Libertarian party } \\
\text { vote Percent from } \\
\text { previous cycle } \\
\text { (same cd) }\end{array}$ & $\begin{array}{l}\text { Historical election } \\
\text { results }\end{array}$ \\
\hline $\begin{array}{l}\text { Lib Vote Percent } \\
\text { Last3 }\end{array}$ & $\mathrm{T}$ & $\mathrm{T}$ & $\mathrm{F}$ & $\begin{array}{l}\text { Libertarian vote } \\
\text { percent from } \\
\text { previous } 3 \text { cycles }\end{array}$ & $\begin{array}{l}\text { Historical election } \\
\text { results }\end{array}$ \\
\hline Median Age & $\mathrm{F}$ & $\mathrm{F}$ & $\mathrm{T}$ & Median age & US Census Bureau \\
\hline Midterm & $\mathrm{F}$ & $\mathrm{T}$ & $\mathrm{F}$ & $\begin{array}{l}\text { Midterm election } \\
\mathrm{T} / \mathrm{F}\end{array}$ & $\begin{array}{l}\text { Historical election } \\
\text { results }\end{array}$ \\
\hline NAOR Involved & $\mathrm{T}$ & $\mathrm{T}$ & $\mathrm{T}$ & $\begin{array}{l}\text { NAOR spent } \\
\text { money T/F }\end{array}$ & $\begin{array}{l}\text { Federal Election } \\
\text { Commission }\end{array}$ \\
\hline NAOR Percent & $\mathrm{T}$ & $\mathrm{T}$ & $\mathrm{T}$ & $\begin{array}{l}\text { Percent of } \\
\text { spending from } \\
\text { NAOR }\end{array}$ & $\begin{array}{l}\text { Federal Election } \\
\text { Commission }\end{array}$ \\
\hline NASDAQ & $\mathrm{F}$ & $\mathrm{F}$ & $\mathrm{T}$ & $\begin{array}{l}\text { NASDAQ } \\
\text { Composite }\end{array}$ & $\begin{array}{l}\text { Federal Reserve } \\
\text { Economic Data }\end{array}$ \\
\hline National Polls & $\mathrm{T}$ & $\mathrm{T}$ & $\mathrm{T}$ & $\begin{array}{l}\text { Average support in } \\
\text { national ballot test } \\
\text { polling }\end{array}$ & Compiled in-house \\
\hline Non-Farm Pay & $\mathrm{F}$ & $\mathrm{F}$ & $\mathrm{T}$ & Nonfarm payrolls & $\begin{array}{l}\text { Federal Reserve } \\
\text { Economic Data }\end{array}$ \\
\hline $\begin{array}{l}\text { Nonhispanic } \\
\text { White Pct }\end{array}$ & $\mathrm{T}$ & $\mathrm{T}$ & $\mathrm{T}$ & $\begin{array}{l}\text { Non-hispanic } \\
\text { white population } \\
\text { percent }\end{array}$ & US Census Bureau \\
\hline NRCC Involved & $\mathrm{T}$ & $\mathrm{F}$ & $\mathrm{F}$ & $\begin{array}{l}\text { NRCC spent } \\
\text { money T/F }\end{array}$ & $\begin{array}{l}\text { Federal Election } \\
\text { Commission }\end{array}$ \\
\hline
\end{tabular}




\begin{tabular}{|c|c|c|c|c|c|}
\hline NRCC Percent & $\mathrm{T}$ & $\mathrm{F}$ & $\mathrm{F}$ & $\begin{array}{l}\text { Percent of } \\
\text { spending from } \\
\text { NRCC }\end{array}$ & $\begin{array}{l}\text { Federal Election } \\
\text { Commission }\end{array}$ \\
\hline Of Prespty & $\mathrm{T}$ & $\mathrm{T}$ & $\mathrm{F}$ & $\begin{array}{l}\text { If GOP candidate } \\
\text { is of the president's } \\
\text { party }\end{array}$ & $\begin{array}{l}\text { Historical election } \\
\text { results }\end{array}$ \\
\hline $\begin{array}{l}\text { Of Prespty By } \\
\text { Midterm }\end{array}$ & $\mathrm{T}$ & $\mathrm{T}$ & $\mathrm{F}$ & $\begin{array}{l}1 \text { = GOP candidate } \\
\text { is of sitting } \\
\text { president's party } \\
\text { and it is a midterm } \\
\text { election year, } 2 \text { = } \\
\text { pres party and } \\
\text { presidential } \\
\text { election year, } 3 \text { = } \\
\text { not pres party and } \\
\text { midterm, } 4=\text { not } \\
\text { pres party and pres } \\
\text { year }\end{array}$ & $\begin{array}{l}\text { Calculated in- } \\
\text { house }\end{array}$ \\
\hline Open Seat & $\mathrm{T}$ & $\mathrm{T}$ & $\mathrm{F}$ & $\begin{array}{l}\text { Indicates whether } \\
\text { this is an open seat } \\
\text { election (current } \\
\text { election) }\end{array}$ & $\begin{array}{l}\text { Historical election } \\
\text { results }\end{array}$ \\
\hline Over 20 Million & $\mathrm{F}$ & $\mathrm{T}$ & $\mathrm{T}$ & $\begin{array}{l}\text { Indicates whether } \\
20 \text { million total } \\
\text { was spent by all } \\
\text { candidates } \\
\text { combined }\end{array}$ & $\begin{array}{l}\text { Federal Election } \\
\text { Commission }\end{array}$ \\
\hline Over 3 Million & $\mathrm{T}$ & $\mathrm{F}$ & $\mathrm{T}$ & $\begin{array}{l}\text { Indicates whether } \\
3 \text { million total was } \\
\text { spent by all } \\
\text { candidates } \\
\text { combined }\end{array}$ & $\begin{array}{l}\text { Federal Election } \\
\text { Commission }\end{array}$ \\
\hline Per Capita Income & $\mathrm{F}$ & $\mathrm{F}$ & $\mathrm{T}$ & $\begin{array}{l}\text { Per capita personal } \\
\text { income }\end{array}$ & $\begin{array}{l}\text { Federal Reserve } \\
\text { Economic Data }\end{array}$ \\
\hline
\end{tabular}




\begin{tabular}{|c|c|c|c|c|c|}
\hline $\begin{array}{l}\text { Personal } \\
\text { Consumption } \\
\text { Expenditures }\end{array}$ & $\mathrm{F}$ & $\mathrm{F}$ & $\mathrm{T}$ & $\begin{array}{l}\text { Personal } \\
\text { consumption } \\
\text { expenditures }\end{array}$ & $\begin{array}{l}\text { Federal Reserve } \\
\text { Economic Data }\end{array}$ \\
\hline Pop Density & $\mathrm{T}$ & $\mathrm{F}$ & $\mathrm{T}$ & $\begin{array}{l}\text { Population density } \\
\text { of a cd/state }\end{array}$ & US Census Bureau \\
\hline Pres By Midterm & F & $\mathrm{T}$ & $\mathrm{F}$ & $\begin{array}{l}\text { Gives party of } \\
\text { sitting president } \\
\text { and indicates } \\
\text { whether election } \\
\text { cycle is midterm } \\
\text { (e.g. "R1" if } \\
\text { Republican } \\
\text { president and } \\
\text { midterm election } \\
\text { year; "D0" if } \\
\text { Democratic } \\
\text { president and not a } \\
\text { midterm election } \\
\text { year) }\end{array}$ & $\begin{array}{l}\text { Calculated in- } \\
\text { house }\end{array}$ \\
\hline Prespty & $\mathrm{T}$ & $\mathrm{T}$ & $\mathrm{F}$ & $\begin{array}{l}\text { Party of current } \\
\text { president }\end{array}$ & $\begin{array}{l}\text { Historical election } \\
\text { results }\end{array}$ \\
\hline Previous Party & $\mathrm{T}$ & $\mathrm{T}$ & $\mathrm{F}$ & $\begin{array}{l}\text { Names the party } \\
\text { that previously } \\
\text { held the seat }\end{array}$ & $\begin{array}{l}\text { Historical election } \\
\text { results }\end{array}$ \\
\hline Primary HHI & $\mathrm{T}$ & $\mathrm{T}$ & $\mathrm{F}$ & $\begin{array}{l}\text { HHI using primary } \\
\text { voters in the } \\
\text { dem+GOP primary } \\
\text { combined }\end{array}$ & $\begin{array}{l}\text { Calculated in- } \\
\text { house }\end{array}$ \\
\hline PVI & $\mathrm{T}$ & $\mathrm{T}$ & $\mathrm{T}$ & $\begin{array}{l}\text { Cook Partisan } \\
\text { Voting Index } \\
\text { (positive values are } \\
\text { R+, negative are } \\
\text { D+) }\end{array}$ & $\begin{array}{l}\text { Calculated in- } \\
\text { house based on } \\
\text { Cook formula }\end{array}$ \\
\hline
\end{tabular}




\begin{tabular}{|c|c|c|c|c|c|}
\hline $\begin{array}{l}\text { PVI Adjusted Net } \\
\text { Approval }\end{array}$ & $\mathrm{F}$ & $\mathrm{F}$ & $\mathrm{T}$ & $\begin{array}{l}\text { PVI minus net } \\
\text { approval for } \\
\text { Democratic } \\
\text { president, plus net } \\
\text { approval for } \\
\text { Republican } \\
\text { president }\end{array}$ & $\begin{array}{l}\text { Calculated in- } \\
\text { house }\end{array}$ \\
\hline R 2 Party Pct & $\mathrm{F}$ & $\mathrm{F}$ & $\mathrm{T}$ & $\begin{array}{l}\text { Republican } \\
\text { percentage of two- } \\
\text { party vote }\end{array}$ & $\begin{array}{l}\text { Historical election } \\
\text { results }\end{array}$ \\
\hline $\begin{array}{l}\text { R Candidate } \\
\text { Ideology }\end{array}$ & $\mathrm{F}$ & $\mathrm{F}$ & $\mathrm{T}$ & $\begin{array}{l}\text { Republican } \\
\text { candidate ideology }\end{array}$ & $\begin{array}{l}\text { Database on } \\
\text { Ideology, Money } \\
\text { in Politics, and } \\
\text { Elections }\end{array}$ \\
\hline $\begin{array}{l}\text { R Consecutive } \\
\text { Terms }\end{array}$ & $\mathrm{F}$ & $\mathrm{F}$ & $\mathrm{T}$ & $\begin{array}{l}\text { Number of } \\
\text { consecutive } \\
\text { Republican terms }\end{array}$ & $\begin{array}{l}\text { Historical election } \\
\text { results }\end{array}$ \\
\hline R Home State & $\mathrm{F}$ & $\mathrm{F}$ & $\mathrm{T}$ & $\begin{array}{l}\text { Home state of } \\
\text { Republican } \\
\text { presidential } \\
\text { candidate }\end{array}$ & $\begin{array}{l}\text { Historical election } \\
\text { results }\end{array}$ \\
\hline R IEM price & $\mathrm{F}$ & $\mathrm{F}$ & $\mathrm{T}$ & $\begin{array}{l}\text { Closing price for } \\
\text { Republican } \\
\text { candidate in } \\
\text { winner-take-all } \\
\text { market on day } \\
\text { before election }\end{array}$ & $\begin{array}{l}\text { Iowa Electronic } \\
\text { Markets }\end{array}$ \\
\hline $\begin{array}{l}\text { R Incumbent } \\
\text { Candidate }\end{array}$ & $\mathrm{F}$ & $\mathrm{F}$ & $\mathrm{T}$ & $\begin{array}{l}\text { Republican } \\
\text { incumbent running }\end{array}$ & $\begin{array}{l}\text { Historical election } \\
\text { results }\end{array}$ \\
\hline R Incumbent Party & $\mathrm{F}$ & $\mathrm{F}$ & $\mathrm{T}$ & $\begin{array}{l}\text { Republican } \\
\text { incumbent running }\end{array}$ & $\begin{array}{l}\text { Historical election } \\
\text { results }\end{array}$ \\
\hline R Overall Pct & $\mathrm{F}$ & $\mathrm{F}$ & $\mathrm{T}$ & $\begin{array}{l}\text { Republican } \\
\text { percentage of } \\
\text { overall vote }\end{array}$ & $\begin{array}{l}\text { Historical election } \\
\text { results }\end{array}$ \\
\hline
\end{tabular}




\begin{tabular}{|c|c|c|c|c|c|}
\hline $\begin{array}{l}\text { R President Net } \\
\text { Approval }\end{array}$ & $\mathrm{F}$ & $\mathrm{F}$ & $\mathrm{T}$ & $\begin{array}{l}\text { Net approval rating } \\
\text { for Republican } \\
\text { president }\end{array}$ & $\begin{array}{l}\text { The American } \\
\text { Presidency Project }\end{array}$ \\
\hline R Primary Margin & $\mathrm{F}$ & $\mathrm{F}$ & $\mathrm{T}$ & $\begin{array}{l}\text { Difference in } \\
\text { overall primary } \\
\text { popular vote } \\
\text { percentage } \\
\text { between } \\
\text { Republican } \\
\text { nominee and } \\
\text { closest primary } \\
\text { challenger }\end{array}$ & $\begin{array}{l}\text { Historical election } \\
\text { results }\end{array}$ \\
\hline R VP Home State & $\mathrm{F}$ & $\mathrm{F}$ & $\mathrm{T}$ & $\begin{array}{l}\text { Home state of } \\
\text { Republican vice } \\
\text { presidential } \\
\text { candidate }\end{array}$ & $\begin{array}{l}\text { Historical election } \\
\text { results }\end{array}$ \\
\hline R Win & $\mathrm{F}$ & $\mathrm{F}$ & $\mathrm{T}$ & Republican win & $\begin{array}{l}\text { Historical election } \\
\text { results }\end{array}$ \\
\hline Race ID & $\mathrm{T}$ & $\mathrm{T}$ & $\mathrm{T}$ & $\begin{array}{l}\text { Unique identifier } \\
\text { reflecting office, } \\
\text { year, state, } \\
\text { district/class }\end{array}$ & $\mathrm{n} / \mathrm{a}$ \\
\hline $\begin{array}{l}\text { Real Personal } \\
\text { Income }\end{array}$ & $\mathrm{F}$ & $\mathrm{F}$ & $\mathrm{T}$ & $\begin{array}{l}\text { Real personal } \\
\text { income }\end{array}$ & $\begin{array}{l}\text { Federal Reserve } \\
\text { Economic Data }\end{array}$ \\
\hline Redistricted & $\mathrm{T}$ & $\mathrm{F}$ & $\mathrm{F}$ & $\begin{array}{l}\text { Indicates } \\
\text { redistricting since } \\
\text { last election }\end{array}$ & $\begin{array}{l}\text { Calculated in- } \\
\text { house }\end{array}$ \\
\hline $\begin{array}{l}\text { Republican } \\
\text { Gender }\end{array}$ & $\mathrm{T}$ & $\mathrm{T}$ & $\mathrm{F}$ & $\begin{array}{l}\text { Gender of GOP } \\
\text { candidate }\end{array}$ & $\begin{array}{l}\text { Database on } \\
\text { Ideology, Money } \\
\text { in Politics, and } \\
\text { Elections }\end{array}$ \\
\hline
\end{tabular}




\begin{tabular}{|c|c|c|c|c|c|}
\hline $\begin{array}{l}\text { Republican } \\
\text { Ideology Score }\end{array}$ & $\mathrm{T}$ & $\mathrm{T}$ & $\mathrm{F}$ & $\begin{array}{l}\text { Ideal point } \\
\text { estimate of } \\
\text { Republican } \\
\text { candidate ideology } \\
\text { based on campaign } \\
\text { finance records } \\
\text { (positive values are } \\
\text { more conservative, } \\
\text { negative values are } \\
\text { more liberal; the } \\
\text { further away from } \\
0 \text { a value is, the } \\
\text { more extreme their } \\
\text { ideology) }\end{array}$ & $\begin{array}{l}\text { Database on } \\
\text { Ideology, Money } \\
\text { in Politics, and } \\
\text { Elections }\end{array}$ \\
\hline State & $\mathrm{T}$ & $\mathrm{T}$ & $\mathrm{T}$ & $\begin{array}{l}\text { State the election is } \\
\text { being held in }\end{array}$ & $\begin{array}{l}\text { Historical election } \\
\text { results }\end{array}$ \\
\hline State Ideology & F & $\mathrm{F}$ & $\mathrm{T}$ & $\begin{array}{l}\text { State/district } \\
\text { ideology }\end{array}$ & $\begin{array}{l}\text { American Ideology } \\
\text { Project }\end{array}$ \\
\hline State Polls & $\mathrm{T}$ & $\mathrm{T}$ & $\mathrm{T}$ & $\begin{array}{l}\text { Average support in } \\
\text { state-level ballot } \\
\text { test polling }\end{array}$ & Compiled in-house \\
\hline $\begin{array}{l}\text { Total Money in } \\
\text { Race }\end{array}$ & $\mathrm{T}$ & $\mathrm{T}$ & $\mathrm{T}$ & $\begin{array}{l}\text { Total money spent } \\
\text { by GOP and Dem }\end{array}$ & $\begin{array}{l}\text { Federal Election } \\
\text { Commission }\end{array}$ \\
\hline $\begin{array}{l}\text { Turnout Count } \\
\text { Last }\end{array}$ & F & $\mathrm{F}$ & $\mathrm{F}$ & $\begin{array}{l}\text { Total turnout from } \\
\text { previous cycle }\end{array}$ & $\begin{array}{l}\text { Historical election } \\
\text { results }\end{array}$ \\
\hline $\begin{array}{l}\text { Turnout Count } \\
\text { Last3 }\end{array}$ & $\mathrm{T}$ & $\mathrm{T}$ & $\mathrm{F}$ & $\begin{array}{l}\text { Total voter turnout } \\
\text { for last } 3 \text { cycles }\end{array}$ & $\begin{array}{l}\text { Historical election } \\
\text { results }\end{array}$ \\
\hline $\begin{array}{l}\text { Unemployment } \\
\text { Rate }\end{array}$ & $\mathrm{T}$ & $\mathrm{T}$ & $\mathrm{T}$ & $\begin{array}{l}\text { Unemployment } \\
\text { rate }\end{array}$ & $\begin{array}{l}\text { Federal Reserve } \\
\text { Economic Data }\end{array}$ \\
\hline $\begin{array}{l}\text { Unemployment } \\
\text { Rate Net Change }\end{array}$ & $\mathrm{T}$ & $\mathrm{T}$ & $\mathrm{F}$ & $\begin{array}{l}\text { State } \\
\text { unemployment rate } \\
\text { net change over } \\
\text { year }\end{array}$ & $\begin{array}{l}\text { Federal Reserve } \\
\text { Economic Data }\end{array}$ \\
\hline
\end{tabular}




\begin{tabular}{|c|c|c|c|c|c|}
\hline $\begin{array}{l}\text { Unemployment } \\
\text { Rate Percent } \\
\text { Change }\end{array}$ & $\mathrm{T}$ & $\mathrm{T}$ & $\mathrm{F}$ & $\begin{array}{l}\text { State } \\
\text { unemployment rate } \\
\text { percent change } \\
\text { over year }\end{array}$ & $\begin{array}{l}\text { Federal Reserve } \\
\text { Economic Data }\end{array}$ \\
\hline $\begin{array}{l}\text { Unopposed } \\
\text { Democrat }\end{array}$ & $\mathrm{T}$ & $\mathrm{F}$ & $\mathrm{F}$ & $\begin{array}{l}\text { Whether dem is } \\
\text { unopposed in this } \\
\text { election }\end{array}$ & $\begin{array}{l}\text { Historical election } \\
\text { results }\end{array}$ \\
\hline $\begin{array}{l}\text { Unopposed } \\
\text { Democrat Last } \\
\text { Cycle }\end{array}$ & $\mathrm{T}$ & $\mathrm{F}$ & $\mathrm{F}$ & $\begin{array}{l}\text { Whether dem ran } \\
\text { unopposed in } \\
\text { previous election }\end{array}$ & $\begin{array}{l}\text { Historical election } \\
\text { results }\end{array}$ \\
\hline $\begin{array}{l}\text { Unopposed } \\
\text { Republican }\end{array}$ & $\mathrm{T}$ & $\mathrm{F}$ & $\mathrm{F}$ & $\begin{array}{l}\text { Whether GOP is } \\
\text { unopposed in this } \\
\text { election }\end{array}$ & $\begin{array}{l}\text { Historical election } \\
\text { results }\end{array}$ \\
\hline $\begin{array}{l}\text { Unopposed } \\
\text { Republican Last } \\
\text { Cycle }\end{array}$ & $\mathrm{T}$ & $\mathrm{F}$ & $\mathrm{F}$ & $\begin{array}{l}\text { Whether GOP ran } \\
\text { unopposed in } \\
\text { previous election }\end{array}$ & $\begin{array}{l}\text { Historical election } \\
\text { results }\end{array}$ \\
\hline Urban Pop Density & $\mathrm{F}$ & $\mathrm{F}$ & $\mathrm{T}$ & $\begin{array}{l}\text { Percent of } \\
\text { population in } \\
\text { urban areas }\end{array}$ & US Census Bureau \\
\hline $\begin{array}{l}\text { Urban Population } \\
\text { Percent }\end{array}$ & $\mathrm{T}$ & $\mathrm{T}$ & $\mathrm{F}$ & $\begin{array}{l}\text { Percent urban } \\
\text { population }\end{array}$ & US Census Bureau \\
\hline Year & $\mathrm{T}$ & $\mathrm{T}$ & $\mathrm{T}$ & $\begin{array}{l}\text { Calendar year } \\
\text { election occurs } \\
\text { within }\end{array}$ & $\begin{array}{l}\text { Historical election } \\
\text { results }\end{array}$ \\
\hline
\end{tabular}

\section{References}

Abramowitz, A. I. (1975). Name familiarity, reputation, and the incumbency effect in a congressional election. Western Political Quarterly, 28(4), 668-684. https://doi.org/10.2307/447984

Barrut, B., \& Schofield N. (2016). Measuring campaign spending effects in post-citizens united congressional elections. In The Political Economy of Social Choices (pp. 205-232). https://doi.org/10.1007/978-3-319-40118$\underline{89}$ 
Brady, D. W., D’Onofrio, R., \& Fiorina, M. P. (2000). The nationalization of electoral forces revisited. In D. W. Brady, J. F. Cogan, \& M. P. Fiorina (Eds.), Continuity and Change in House Elections (pp. 130-148).

Campbell, J. E. (2010). The seats in trouble forecast of the 2010 elections to the US House. PS: Political Science \& Politics, 43(4), 627-630. https://doi.org/10.1017:S1049096510001095

Edwards, G. C. (2009). Presidential approval as a source of influence in Congress. Oxford Handbook of the American Presidency. https://doi.org/10.1093/oxfordhb/9780199238859.003.0015

Erikson, R. S. (1971). The advantage of incumbency in congressional elections. Polity, 3(3), 395-405. https://doi.org/10.2307/3234117

Erikson, R. S. (1988). The puzzle of midterm loss. The Journal of Politics, 50(4), 1011-1029. https://doi.org/10.2307/2131389

Green, D., \& Gerber, A.S. (2006). Can registration-based sampling improve the accuracy of midterm forecasts? Public Opinion Quarterly, 70(2), 197-223. https://doi.org/10.1093/poq/nfju22

Jacobson, G. C. (1978). The effects of campaign spending in congressional elections. American Political Science Review, 72(2), 469-491. https://doi.org/10.2307/1954105

Lewis-Beck, M. S., \& Rice, T. W. (1984). Forecasting U.S. House elections. Legislative Studies Quarterly, 9, 475-486. https://doi.org/10.2307/439492

Lewis-Beck, M. S., \& Tien, C. (2014). Congressional election forecasting: structure-X models for 2014. PS: Political Science \& Politics, 47(4), 782-785. https://doi.org/10.1017/S1049096514001267

Montgomery, J. M., Hollenbach, F. M., \& Ward, M. D. (2012). Improving predictions using ensemble Bayesian model averaging. Political Analysis, 20(3), 271-291. https://doi.org/10.1093/pan/mps002

Pedregosa, F., Varoquaux, G., Gramfort, A., Michel, V., Thirion, B., Grisel, O., Blondel, M., Prettenhofer, P., Weiss, R., Dubourg, V. \& Vanderplas, J. (2011). Scikit-learn: Machine learning in Python. The Journal of Machine Learning Research, 12(85), 2825-2830. https://www.jmlr.org/papers/v12/pedregosa11a.html

Shirani-Mehr, H., Rothschild, D., Goel, S., \& Gelman, A. (2018). Disentangling bias and variance in election polls. Journal of the American Statistical Association, 113(522), 607-614.

https://doi.org/10.1080/01621429.2018.1448823

Stokes, D. E., \& Miller, W. E. (1962). Party government and the saliency of Congress. Public Opinion Quarterly, 26(4), 531-546. https://doi-org.eres.qnl.qa/10.1086/267126 
Tufte, E. R. (1975). Determinants of the outcomes of midterm congressional elections. American Political Science Review, 69(3), 812-826. https://doi.org/10.2307/1958391

Zou, H., \& Hastie, T. (2005). Regularization and variable selection via the elastic net. Journal of the royal statistical society: series B (statistical methodology), 67(2), 301-320. https://doi.org/10.1111/j.14679868.2005.00503.x

(C2020 Kiel Williams, Mukul Ram, Matthew Shor, Sreevani Jarugula, Dan DeRemigi, Alex Alduncin, and Scott Tranter. This article is licensed under a Creative Commons Attribution (CC BY 4.0) International license, except where otherwise indicated with respect to particular material included in the article.

\section{Footnotes}

1. The unit of analysis in the presidential model is state equivalents (SEs), which includes 50 states, the District of Columbia, and each congressional district from both Maine and Nebraska due to the rules surrounding the allocation of those states' electoral votes. $ヒ$

2. A full list of variables is included in the appendix. $ヒ$

3. A previous version of this post-2018 performance analysis was publicly released in November 2018 and

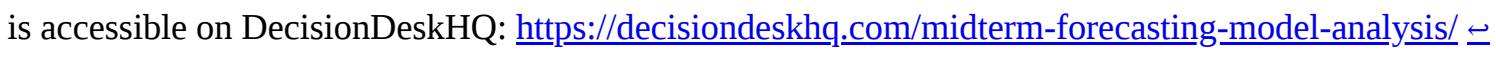

4. NC-11 results were invalidated due to fraud and are excluded for the purpose of this analysis. $€$ 